Research Article

\title{
Fluid-Induced Vibration Elimination of a Rotor/Seal System with the Dynamic Vibration Absorber
}

\author{
Qi Xu (D), ${ }^{1}$ Junkai Niu $\left(\mathbb{D},{ }^{1}\right.$ Hongliang Yao $\mathbb{D}^{2},{ }^{2}$ Lichao Zhao $\mathbb{D}^{3},{ }^{3}$ and Bangchun Wen ${ }^{2}$ \\ ${ }^{1}$ School of Mechanical Engineering, Shenyang University of Technology, Shenyang 110870, China \\ ${ }^{2}$ School of Mechanical Engineering and Automation, Northeastern University, Shenyang 110819, China \\ ${ }^{3}$ Installation Accessories Co. Ltd., Shenyang Blower Group Co. Ltd., Shenyang 110869, China \\ Correspondence should be addressed to Hongliang Yao; hlyao@mail.neu.edu.cn
}

Received 16 August 2018; Revised 11 October 2018; Accepted 6 November 2018; Published 2 December 2018

Academic Editor: Gabriele Cazzulani

Copyright ( 2018 Qi Xu et al. This is an open access article distributed under the Creative Commons Attribution License, which permits unrestricted use, distribution, and reproduction in any medium, provided the original work is properly cited.

\begin{abstract}
The dynamic vibration absorbers have been applied to attenuate the rotor unbalance and torsional vibrations. The major purpose of this paper is to research the elimination of the fluid-induced vibration in the rotor/seal system using the absorber. The simplified rotor model with the absorber is established, and the Muszynska fluid force model is employed for the nonlinear seal force. The numerical method is used for the solutions of the nonlinear differential equations. The nonlinear responses of the rotor/seal system without and with the absorber are obtained, and then the rotating speed ranges by which the fluid-induced instability can be eliminated completely and partially are presented, respectively. The absorber parameters ranges by which the instability vibration can be eliminated completely and partially are obtained. The results show that the natural frequency vibration due to the fluid-induced instability in the rotor/seal system can be eliminated efficiently using the absorber. The appropriate natural frequency and damping ratio of the absorber can extend the complete elimination region of the instability vibration and postpone the occurrence of the instability vibration.
\end{abstract}

\section{Introduction}

Too large fluid-induced vibration in the turbomachinery is unacceptable as it may lead to shut down quickly of the rotating machinery and then causes huge economic loss [1]. The instability vibration generates by the oil film force in the journal bearing and the seal force between the rotor and seal. The clearance filled with the fluid between rotor and stator (bearing or seal) is small. The pressure difference arises in the clearance by some unavoidable reasons, such as the elastic deformation of the shaft and the rotor unbalance. Then, the fluid-induced force acts on the rotor system. The instability vibration appears when the system damping is insufficient.

The methods to eliminate the instability vibration are divided into two kinds: the parameters adjustment of the fluid force and the vibration control by applying the external force. The antiswirl injection was a typical method to eliminate the instability vibration by disrupting the fluid flow based on the injection of an additional flow to the seal, in the tangential direction, opposite to the direction of the shaft rotation by Muszynska and Bently [2]. Le et al. [3] proposed that the optimally controlled antiswirl injection method was applied to eliminate the whirl in the fluid-film bearings of the rotary machinery using the linear quadratic regulator method. Sun et al. [4] designed four kinds of the seals with and without the antiswirl flow. The theoretical analysis and experimental research on the seal performances of the antiswirl flow showed that the antiswirl flow could effectively reduce the effective stiffness and increase the effective damping. The seal configuration such as the annular honeycomb seal was another typical method to eliminate the instability vibration by disrupting the fluid flow in the seal by Childs et al. [5]. Subramanian et al. [6] performed both centrifugal and thermal growth on the leakage characteristics of the labyrinth seal in the gas turbine. Ertas et al. [7] compared the rotordynamic performance for three types of seals, which include the labyrinth, honeycomb, and fully 
partitioned damper seals. The instability vibration induced by the journal bearing is in general prevented using the multilobed and tilting-pad journal bearing. Chasalevris and Dohnal [8] presented the adjustable journal bearings to extend the stability margins of the rotor-fluid film journal bearings system for the journal bearing-induced instabilities. The proposed 2-arc and 3-arc journal bearing configurations could provide the periodical stiffness and damping variation. Salazar and Santos [9-11] presented the model of the flexible rotor-active tilting-pad journal bearing system and designed and implemented the model-based controller for the system, to reduce the amplitude of the frequency response at resonance. The instability vibration is also suppressed by controlling the flow or pressure in the seal or bearing. Queiroz [12] proposed the active hydrodynamic bearing to mitigate such vibration in the lightly loaded rotating machines. A rotating bushing by which the speed could be controlled was added in the bearing. The PID-like feedback control law for the bushing speed was proposed and tested via the numerical simulations. Applying the external force is another kind of the method to eliminate the instability vibration. Fan and Pan [13] presented the active electromagnetic actuator to raise the threshold of instability of the rotor-fluid film bearings and eliminate both oil and dry whips instabilities in the normal, operational range. The magnetic actuator was applied to control the oil whip instability vibration using the $\mu$-synthesis control technique, as shown by Riemann et al. [14].

The dynamic vibration absorbers (DVAs), as a kind of the method for eliminating the vibration, have been widely applied in the structure vibration suppression. Parseh et al. and Chen et al. $[15,16]$ presented the nonlinear energy sink (NES) to suppress the vibration of the linear beam. Mamaghani et al. [17] presented the NESs to control the vibration of the pipe conveying the fluid under the external periodic excitation. Ebrahimzade et al. [18] investigated the stability properties and nonlinear behaviours of the wing model attached with the DVA and NES. Recently, the DVAs have also been applied in the rotor system. The unbalance and torsional vibrations have been attenuated using the DVAs. Yao et al. [19, 20] proposed the negative stiffness DVA and magnetic NES to attenuate the unbalance vibration in the rotor system. The negative stiffness DVA and magnetic NES had better performance over a wider frequency range than the linear DVA. Hu and $\mathrm{He}$ [21] presented the rotor DVAs to online control the critical speed vibration of the single-span rotor system. The effect of the installation position of the rotor DVAs on the vibration control performance was studied. Bab et al. [22] investigated the vibration attenuation of the smooth NESs for the rotor system with mass eccentricity. They also investigated the vibration mitigation ability of the smooth NESs for the rotor system supported by journal bearings with the nonlinear suspensions [23]. After that, they located the NESs on the disk and bearing to investigate the effects of the NES on the vibration mitigation in the continuous rotor-disk-journal bearing system with the nonlinear suspensions [24]. Guo et al. [25] presented the vibration reduction in the unbalanced hollow rotor system using the NESs.
Besides, the centrifugal pendulum vibration absorbers (CPVAs), as another typical kind of the DVA, have long been employed in the torsional vibration attenuation in the rotating and reciprocating machines [26]. Haddow and Shaw [27] proposed the dynamic behaviour of the torsional vibration absorbers by the experimental and theoretical investigation. Issa and Shaw [28] presented the equivalent translational system model of the rotor fitted with the CPVAs to investigate the nonlinear dynamic responses of the equivalent translational system. The localization phenomena of the nonlinear torsional vibration suppression in the rotating shaft system attached with the CPVAs were investigated by Nishimura et al. [29]. Vidmar et al. [30] presented the effects of the coulomb friction on the performance of the rotor-CPVAs system by the perturbation analysis and experimental measurements. Shi and Parker [31] investigated the natural frequencies, vibration modes, and stability of the CPVAs system with the equally spaced, identical absorbers by the analytical model of the rotorCPVAs system. Then, the symmetry breaking effects on the modal properties of the CPVAs systems attached with the multiple groups of the absorbers were discussed [32]. After that, they investigated the vibration reduction in the rigid rotor with the tilting, rotational, and translational motions using the CPVAs [33].

The vibration attenuation for the rotor using the DVAs has been verified by plenty references. However, the research on the method of the vibration attenuation using the DVAs for the rotor system is mainly focused on the unbalance and torsional vibration. To the authors' knowledge, few papers are devoted to the fluid-induced instability vibration elimination for the rotor/seal system using the DVAs. So, the fluidinduced vibration elimination of the rotor/seal system with the DVA is proposed in this paper. Firstly, the rotor/seal system model attached with the DVA is established by the simplified rotor and Muszynska seal force model. Secondly, the nonlinear responses of the rotor/seal system without and with the DVA are studied using the numerical method. Thirdly, the influences of the natural frequency and damping ratio of the DVA on the fluid-induced vibration elimination are investigated. Finally, some conclusions are drawn to summarize the effects of fluid-induced vibration elimination for the rotor/seal system by the DVA.

\section{Model of Rotor/Seal System with DVA}

2.1. Structure of Rotor/Seal System with DVA. The schematic diagram of the structure of the rotor/seal system with the DVA is shown in Figure 1. A single disk rotor is supported rigidly. The rotor/seal system is a two-lateral-mode isotropic rotor with the fluid interaction in the rotor-to-stationary part clearances. The Muszynska fluid force model is used for the nonlinear seal force $[2,34]$ and equivalently acts on the disk. The DVA is attached on the shaft by the rolling bearing with the frame [19-21], so it does not rotate with the rotor. The DVA is added in the horizontal and vertical directions, respectively. The spring and damping elements are connected to the supporting frame of the rolling bearing. To stop the rotating of the DVA, the flexible connections exist between the DVA and the rotor 


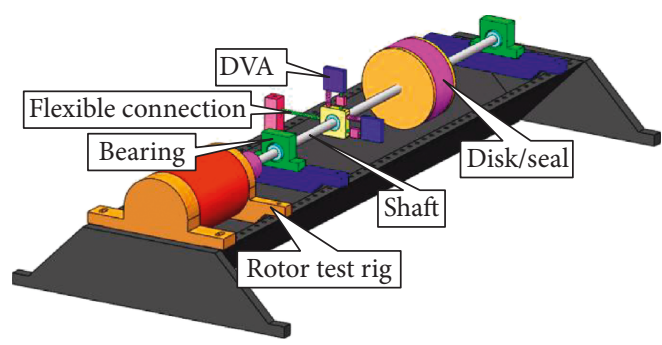

(a)

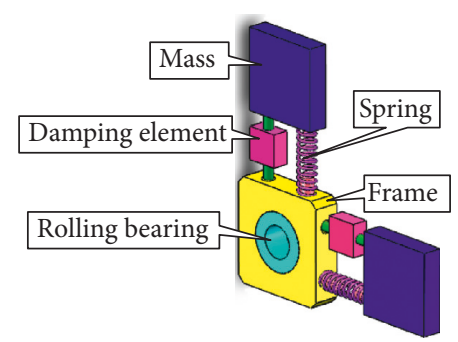

(b)

Figure 1: The schematic diagram of the structure of the rotor/seal system with the DVA. (a) The structure of the whole test rig. (b) The structure of the DVA.

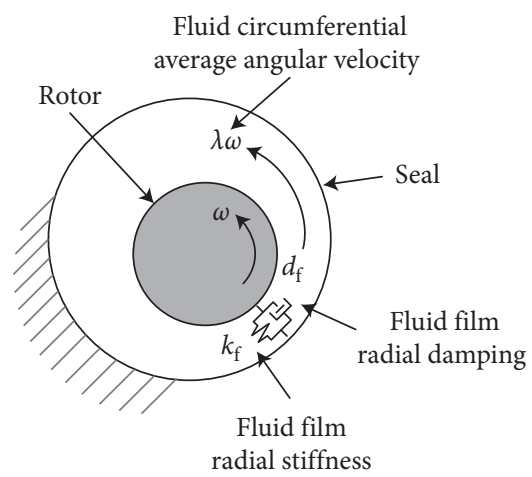

(a)

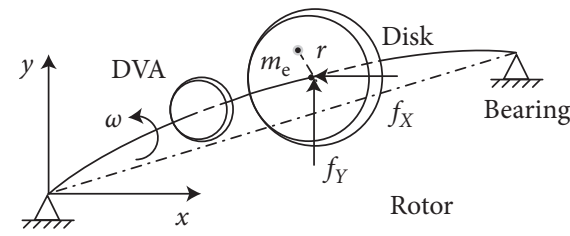

(b)

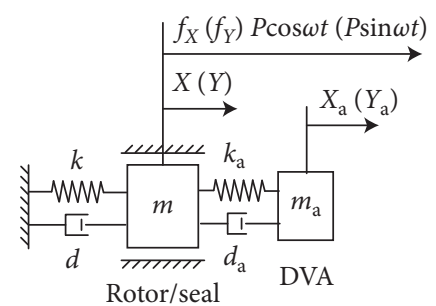

(c)

Figure 2: The model of the rotor/seal system with the DVA. (a) The nonlinear seal force model. (b) The rotor/seal system with the DVA. (c) The equivalent model.

test bench. The flexible connections are springs with very weak stiffness, similar as that of the DVA in References $[19,21]$.

2.2. Dynamic Model of Rotor/Seal System with DVA. The dynamic model of the rotor/seal system with the DVA is shown in Figure 2. Figures 2(a)-2(c) are the schematic diagram of the nonlinear seal force model, the rotor/seal system with the DVA, and the equivalent model. The equations of motion of the system may be expressed as follows:

$$
\left\{\begin{array}{l}
m \ddot{X}+d \dot{X}+k X+f_{X}+d_{\mathrm{a}}\left(\dot{X}-\dot{X}_{\mathrm{a}}\right)+k_{\mathrm{a}}\left(X-X_{\mathrm{a}}\right)=P \cos \omega t, \\
m \ddot{Y}+d \dot{Y}+k Y+f_{Y}+d_{\mathrm{a}}\left(\dot{Y}-\dot{Y}_{\mathrm{a}}\right)+k_{\mathrm{a}}\left(Y-Y_{\mathrm{a}}\right)=P \sin \omega t, \\
m_{\mathrm{a}} \ddot{X}_{\mathrm{a}}+d_{\mathrm{a}}\left(\dot{X}_{\mathrm{a}}-\dot{X}\right)+k_{\mathrm{a}}\left(X_{\mathrm{a}}-X\right)=0, \\
m_{\mathrm{a}} \ddot{Y}_{\mathrm{a}}+d_{\mathrm{a}}\left(\dot{Y}_{\mathrm{a}}-\dot{Y}\right)+k_{\mathrm{a}}\left(Y_{\mathrm{a}}-Y\right)=0,
\end{array}\right.
$$

$\left\{\begin{array}{l}f_{X}=m_{\mathrm{f}} \ddot{X}+d_{\mathrm{f}} \dot{X}+2 m_{\mathrm{f}} \omega \lambda \dot{Y}+\left(k_{\mathrm{f}}-m_{\mathrm{f}} \omega^{2} \lambda^{2}\right) X+\omega d_{\mathrm{f}} \lambda Y, \\ f_{Y}=m_{\mathrm{f}} \ddot{Y}-2 m_{\mathrm{f}} \omega \lambda \dot{X}+d_{\mathrm{f}} \dot{Y}-\omega d_{\mathrm{f}} \lambda X+\left(k_{\mathrm{f}}-m_{\mathrm{f}} \omega^{2} \lambda^{2}\right) Y,\end{array}\right.$

$$
\begin{aligned}
P & =m_{\mathrm{e}} \omega^{2} r, \\
d_{\mathrm{f}} & =d_{0}\left(1-U^{2}\right)^{-n}, \\
k_{\mathrm{f}} & =k_{0}\left(1-U^{2}\right)^{-n}, \\
k_{\mathrm{f}} & =k_{0}\left(1-U^{2}\right)^{-n}, \\
\lambda & =\lambda_{0}(1-U)^{b}, \\
U & =\frac{\sqrt{X^{2}+Y^{2}}}{r_{\mathrm{f}}},
\end{aligned}
$$

where $m, d$, and $k$ denote, respectively, rotor generalized (modal) mass, lateral isotropic stiffness, and lateral external damping. $m_{\mathrm{e}}$ and $r$ are the eccentric mass and eccentricity, respectively. $f_{X}$ and $f_{Y}$ are the nonlinear seal force. $m_{\mathrm{f}}, d_{\mathrm{f}}$, and $k_{\mathrm{f}}$ denote, respectively, the fluid inertia, fluid film radial damping, and stiffness coefficients in the seal. $\lambda$ is the fluid circumferential average velocity ratio. $d_{0}, k_{0}, \lambda_{0}, n$, and $b$ are the parameters of the nonlinear fluid force. These parameters of the nonlinear force are described in detail by Muszynska [34]. $r_{\mathrm{f}}$ is the radial clearance of the seal. $\omega$ is the rotating speed. $m_{\mathrm{a}}, d_{\mathrm{a}}$, and $k_{\mathrm{a}}$ are the mass, damping, and stiffness coefficients of the DVA, respectively. $X, Y$ and $X_{\mathrm{a}}, Y_{\mathrm{a}}$ are the horizontal and vertical displacements of the rotor and the DVA, respectively. $d_{\mathrm{f}}, k_{\mathrm{f}}$, and $\lambda$ are the nonlinear functions of the displacements $X$ and $Y$. 
The stiffness of the flexible connections is neglected since it is very small comparing with $k_{\mathrm{a}}$. So, the stiffness term of the flexible connections is omitted in Equation (1).

The nondimensional transform is as follows:

$$
\begin{gathered}
x=\frac{X}{r_{\mathrm{f}}}, \\
y=\frac{Y}{r_{\mathrm{f}}}, \\
x_{\mathrm{a}}=\frac{X_{\mathrm{a}}}{r_{\mathrm{f}}}, \\
y_{\mathrm{a}}=\frac{Y_{\mathrm{a}}}{r_{\mathrm{f}}}, \\
\tau=\omega t, \\
\frac{d}{d t}=\omega \frac{d}{d \tau}, \\
\frac{d^{2}}{d t^{2}}=\omega^{2} \frac{d^{2}}{d \tau^{2}},
\end{gathered}
$$

and is defined as follows:

$$
\begin{aligned}
& x^{\prime}=\frac{d}{d \tau} x, \\
& y^{\prime}=\frac{d}{d \tau} y, \\
& x^{\prime \prime}=\frac{d^{2}}{d \tau^{2}} x, \\
& y^{\prime \prime}=\frac{d^{2}}{d \tau^{2}} y, \\
& x_{\mathrm{a}}^{\prime}=\frac{d}{d \tau} x_{\mathrm{a}}, \\
& y_{\mathrm{a}}^{\prime}=\frac{d}{d \tau} y_{\mathrm{a}}, \\
& x_{\mathrm{a}}^{\prime \prime}=\frac{d^{2}}{d \tau^{2}} x_{\mathrm{a}}, \\
& y_{\mathrm{a}}^{\prime \prime}=\frac{d^{2}}{d \tau^{2}} y_{\mathrm{a}} .
\end{aligned}
$$

By substituting Equations (4)-(6) into Equations (1)-(3), the original equations become the nondimensional equations:

$$
\begin{aligned}
& \left\{\begin{array}{l}
x^{\prime \prime}+2 \zeta_{\mathrm{r}} \bar{\omega}_{\mathrm{r}} x^{\prime}+\bar{\omega}_{\mathrm{r}}^{2} x+f_{x}+2 \varepsilon \zeta_{\mathrm{a}} \bar{\omega}_{\mathrm{a}}\left(x^{\prime}-x_{\mathrm{a}}^{\prime}\right)+\varepsilon \bar{\omega}_{\mathrm{a}}^{2}\left(x-x_{\mathrm{a}}\right)=\varepsilon_{\mathrm{e}} \cos \tau, \\
y^{\prime \prime}+2 \zeta_{\mathrm{r}} \bar{\omega}_{\mathrm{r}} y^{\prime}+\bar{\omega}_{\mathrm{r}}^{2} y+f_{y}+2 \varepsilon \zeta_{\mathrm{a}} \bar{\omega}_{\mathrm{a}}\left(y^{\prime}-y_{\mathrm{a}}^{\prime}\right)+\varepsilon \bar{\omega}_{\mathrm{a}}^{2}\left(y-y_{\mathrm{a}}\right)=\varepsilon_{\mathrm{e}} \sin \tau, \\
x_{\mathrm{a}}^{\prime \prime}+2 \zeta_{\mathrm{a}} \bar{\omega}_{\mathrm{a}}\left(x_{\mathrm{a}}^{\prime}-x^{\prime}\right)+\bar{\omega}_{\mathrm{a}}^{2}\left(x_{\mathrm{a}}-x\right)=0, \\
y_{\mathrm{a}}^{\prime \prime}+2 \zeta_{\mathrm{a}} \bar{\omega}_{\mathrm{a}}\left(y_{\mathrm{a}}^{\prime}-y^{\prime}\right)+\bar{\omega}_{\mathrm{a}}^{2}\left(y_{\mathrm{a}}-y\right)=0,
\end{array}\right. \\
& \left\{\begin{array}{l}
f_{x}=\varepsilon_{\mathrm{f}} x^{\prime \prime}+\mu_{\mathrm{d}} x^{\prime}+2 \varepsilon_{\mathrm{f}} \lambda y^{\prime}+\left(\mu_{\mathrm{k}}-\varepsilon_{\mathrm{f}} \lambda^{2}\right) x+\mu_{\mathrm{d}} \lambda y, \\
f_{y}=\varepsilon_{\mathrm{f}} y^{\prime \prime}-2 \varepsilon_{\mathrm{f}} \lambda x^{\prime}+\mu_{\mathrm{d}} y^{\prime}-\mu_{\mathrm{d}} \lambda x+\left(\mu_{\mathrm{k}}-\varepsilon_{\mathrm{f}} \lambda^{2}\right) y,
\end{array}\right. \\
& d_{\mathrm{f}}=d_{0}\left(1-u^{2}\right)^{-n} \text {, } \\
& k_{\mathrm{f}}=k_{0}\left(1-u^{2}\right)^{-n} \text {, } \\
& \lambda=\lambda_{0}(1-u)^{b}, \\
& u=\sqrt{x^{2}+y^{2}} \text {, }
\end{aligned}
$$

where $\omega_{\mathrm{r}}=\sqrt{k / m}, \zeta_{\mathrm{r}}=d /\left(2 m \omega_{\mathrm{r}}\right), \varepsilon=m_{\mathrm{a}} / m, \omega_{\mathrm{a}}=\sqrt{k_{\mathrm{a}} / m_{\mathrm{a}}}$, $\zeta_{\mathrm{a}}=d_{\mathrm{a}} /\left(2 m_{\mathrm{a}} \omega_{\mathrm{a}}\right), \quad \varepsilon_{\mathrm{e}}=m_{\mathrm{e}} r /\left(m r_{\mathrm{f}}\right), \quad \bar{\omega}_{\mathrm{r}}=\omega_{\mathrm{r}} / \omega, \quad \bar{\omega}_{\mathrm{a}}=\omega_{\mathrm{a}} / \omega$, $\varepsilon_{\mathrm{f}}=m_{\mathrm{f}} / m, \mu_{\mathrm{d}}=d_{\mathrm{f}} /(m \omega)$, and $\mu_{\mathrm{k}}=k_{\mathrm{f}} /\left(m \omega^{2}\right)$

\section{Numerical Simulations and Discussions}

3.1. Parameters Definition and Numerical Method. The numerical method, Newmark time integration method [35], is used to solve the complicated nonlinear equations and the detailed algorithm is introduced in Appendix. The algorithm is unconditionally convergent as the values of parameters $\beta \geq$ $1 / 4$ and $\delta \geq 1 / 2$ [36]. The parameters of the rotor/seal system are as follows: the rotor mass $m=1 \mathrm{~kg}$, the damping ratio $\zeta_{\mathrm{r}}$
$=0.025$, and the natural frequency $\omega_{\mathrm{r}}=200 \mathrm{rad} / \mathrm{s}$; the radial clearance of the seal $r_{\mathrm{f}}=10^{-3} \mathrm{~m}$; and the nonlinear seal force parameters $\varepsilon_{\mathrm{f}}=10^{-6}, d_{0}=100 \mathrm{Nm} / \mathrm{s}, k_{0}=1 \mathrm{~N} / \mathrm{m}, \lambda_{0}=0.48, n$ $=2$, and $b=0.5$. Besides, $\varepsilon_{\mathrm{e}}$ is set as 0.01 . The nonlinear behaviours of the real rotor/seal system can be described by the above parameters.

3.2. Nonlinear Characteristics of Rotor/Seal System. The bifurcation diagram and the amplitude-frequency response curve of the rotor/seal system in the $x$ coordinate are established, and the results are shown in Figure 3. The rotating speed $\omega$ as a bifurcation parameter is considered. 


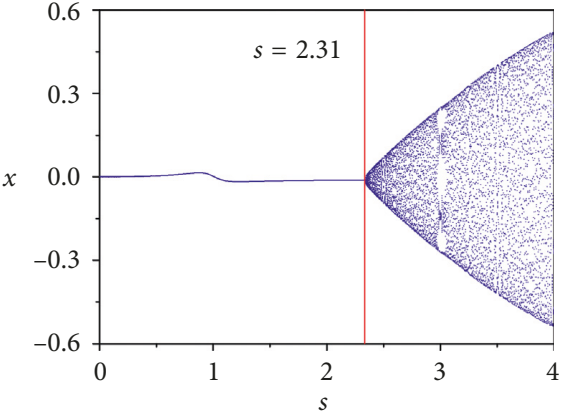

(a)

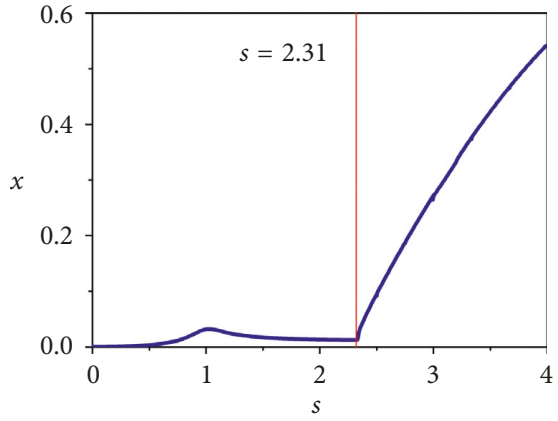

(b)

FIgURE 3: (a) The bifurcation diagram and (b) the amplitude-frequency response curve of the rotor/seal system in the $x$ coordinate.

Assuming the nondimensional rotating speed $s=\omega / \omega_{\mathrm{r}}$. It can be observed in Figure 3(a) that the vibration response is periodic at $s<2.31$. Only one point is correspondingly shown in the bifurcation diagram for every rotating speed. The vibration response is $n$ T periodic or quasiperiodic at $s \geq 2.31$ and can be determined by Poincaré map. The nonlinear terms in the equations are the reason of the vibration instability in the rotor system. $s=2.31$ is the instability threshold of the rotor/seal system. Consequently, the vibration responses of the rotor/seal system in Figure 3(a) can be divided into two regions: the synchronous response at $s<2.31$ and the instability vibration at $s \geq 2.31$. Figure $3(\mathrm{~b})$ shows that the resonance occurs as the rotating speed is close to $s=1$. As the rotating speed exceeds the instability threshold of the rotor/seal system $s=2.31$, the amplitude of the rotor/seal system increases rapidly, which is caused by the fluid-induced instability.

The synchronous response $s=2$ and the instability responses $s=2.35, s=2.5, s=2.65$, and $s=2.8$ are picked, respectively, in the $x$ coordinate for the following analysis as shown in Figures $4-8$. Figure 4 shows that the $1 \mathrm{X}$ frequency only exists in the frequency spectrum, the orbit of the rotor is a regular circle, and an isolated point is in the Poincaré section. These results indicate that, in this case, the rotor has a periodic solution. Figure 5 represents that the $1 \mathrm{X}$ frequency and the natural frequency $\omega_{\mathrm{r}}$ of the rotor components exist in the frequency spectrum, the orbit of the rotor is irregular, and a closed curve formed by the infinite points is in the Poincaré section. These results indicate that, in this case, the rotor has a quasiperiodic solution. Figure 6 represents that the $1 \mathrm{X}$ frequency and the natural frequency $\omega_{\mathrm{r}}$ of the rotor components exist in the frequency spectrum, the orbit of the rotor is irregular, and five isolated points are in the Poincare section. These results indicate that, in this case, the rotor has a $5 \mathrm{~T}$ periodic solution. Figure 7 shows that the $1 \mathrm{X}$ frequency and the natural frequency $\omega_{\mathrm{r}}$ of the rotor components exist in the frequency spectrum, the orbit of the rotor is irregular, and a closed curve formed by the infinite points is in the Poincare section. These results indicate that, in this case, the rotor has also a quasiperiodic solution. Figure 8 shows that the $1 \mathrm{X}$ frequency and the natural frequency $\omega_{\mathrm{r}}$ of the rotor components exist in the frequency spectrum, the orbit of the rotor is irregular, and the fourteen isolated points are in the Poincaré section. These results indicate that, in this case, the rotor has a 14T-periodic solution. Compared with the frequency spectrums of the $x$ coordinate in Figures 4(a), 5(a), 6(a), $7(\mathrm{a})$, and $8(\mathrm{a})$, the amplitude of the 1X frequency is almost constant but that of the natural frequency $\omega_{\mathrm{r}}$ increases as the rotating speed increases.

3.3. Nonlinear Characteristics of Rotor/Seal System with the $D V A$. The main object of the paper is to eliminate the natural frequency vibration due to the fluid-induced instability when the rotating speed exceeds the instability threshold of the rotor/seal system. The DVA is attached on the rotor/seal system. The parameters of the DVA are considered: picking the mass ratio $\varepsilon=0.01$ since the DVA mass ratio is limited for the practical considerations. It is known that the fluid-induced vibration frequency is almost equal to the natural frequency of the rotor system, so the natural frequency of the DVA $\omega_{\mathrm{a}}=\omega_{\mathrm{r}}$ is picked. Normally, the damping ratio is relatively small, so the damping ratio $\zeta_{\mathrm{a}}$ $=0.05$ is picked. The influences of the natural frequency and damping ratio are discussed in the next section. In the case, the rotor-DVA system has two natural frequencies: the firstand second-order natural frequencies $\omega_{n 1}$ and $\omega_{n 2}$, respectively.

Correspondingly, the synchronous response $s=2$ and the instability responses $s=2.35, s=2.5, s=2.65$, and $s=2.8$ are picked, respectively, in the $x$ coordinate for the effect analysis of the instability vibration elimination as shown in Figures 9-13. The blue solid line with the solid circle is the response of the rotor/seal system, and the red solid line with the open circle is the response of the rotor/seal system with the DVA, respectively. Figure 9 shows that the response of the rotor/seal system with the DVA is the same as that of the rotor/seal system without the DVA at $s=2$. Figure 10 represents that the instability vibration of the rotor natural frequency $\omega_{\mathrm{r}}$ is eliminated completely by the DVA and the $1 \mathrm{X}$ frequency has no change in the frequency spectrum, the orbit of the rotor becomes a regular circle, and an isolated point instead of a closed curve formed by the infinite points is in the Poincaré section. The quasiperiodic response becomes the periodic response. Figure 11 


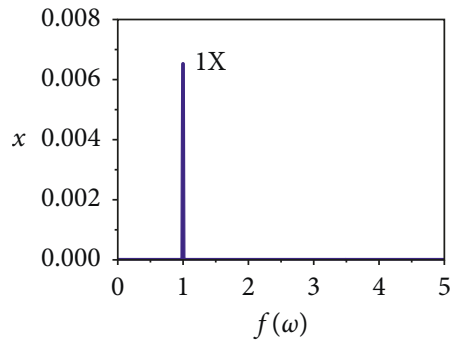

(a)

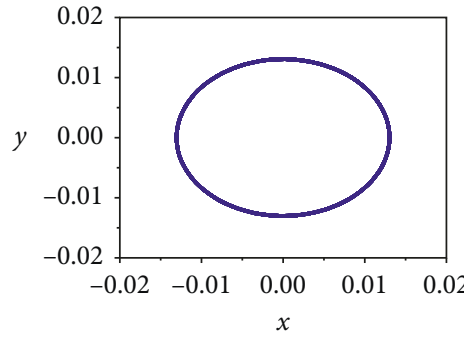

(b)

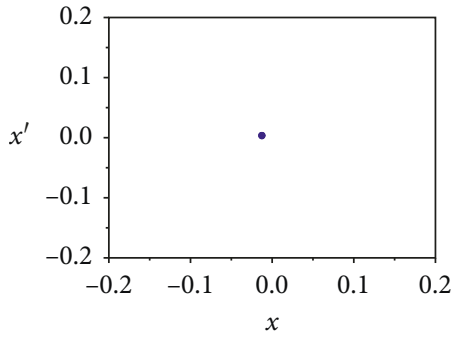

(c)

Figure 4: The periodic response of the rotor/seal system at $s=2$. (a) The frequency spectrum of the $x$ coordinate. (b) The orbit of the rotor. (c) The Poincaré map of the $x$ coordinate.

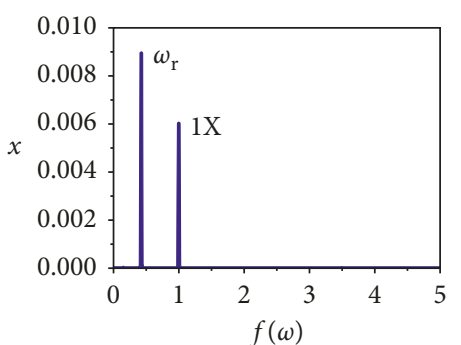

(a)

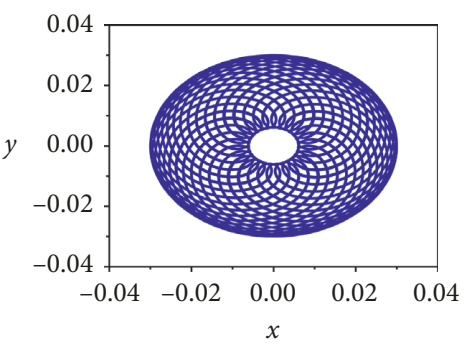

(b)

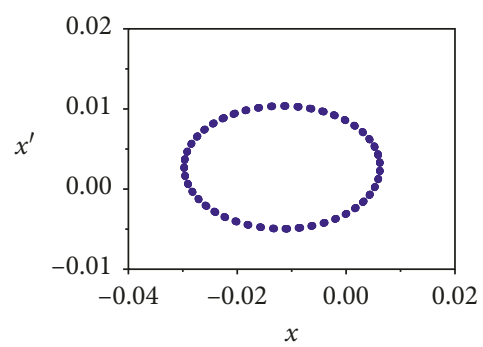

(c)

Figure 5: The quasiperiodic response of the rotor/seal system at $s=2.35$. (a) The frequency spectrum of the $x$ coordinate. (b) The orbit of the rotor. (c) The Poincaré map of the $x$ coordinate.

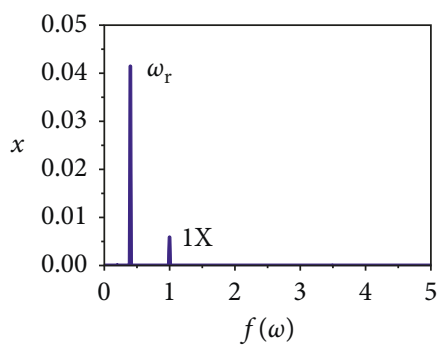

(a)

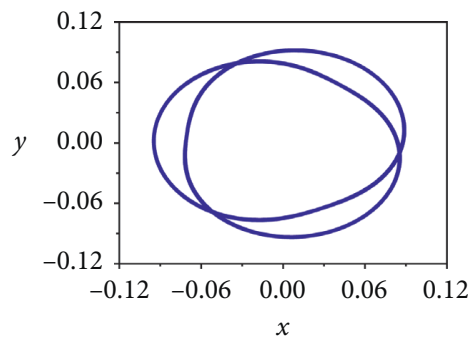

(b)

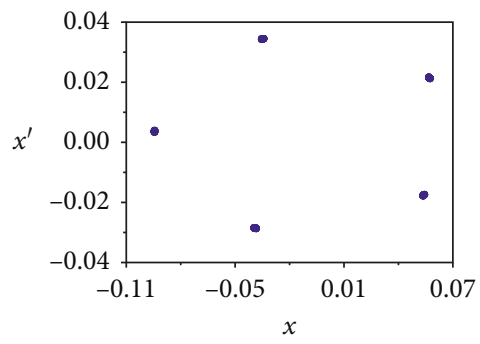

(c)

Figure 6: The 5T-periodic response of the rotor/seal system at $s=2.5$. (a) The frequency spectrum of the $x$ coordinate. (b) The orbit of the rotor. (c) The Poincaré map of the $x$ coordinate.

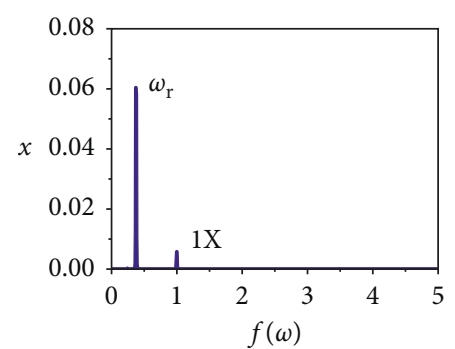

(a)

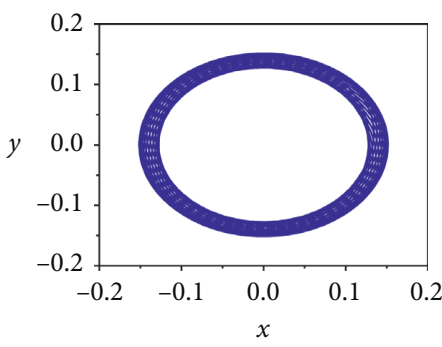

(b)

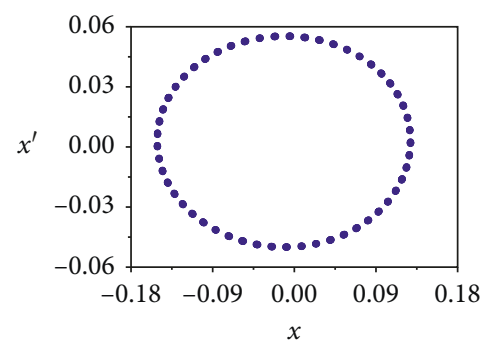

(c)

FIGURE 7: The quasiperiodic response of the rotor/seal system at $s=2.65$. (a) The frequency spectrum of the $x$ coordinate. (b) The orbit of the rotor. (c) The Poincaré map of the $x$ coordinate.

represents that the instability vibration of the rotor natural frequency $\omega_{\mathrm{r}}$ is also eliminated completely by the DVA, and the $1 \mathrm{X}$ frequency has no change in the frequency spectrum, the orbit of the rotor becomes a regular circle, and an isolated point instead of five isolated points is in the Poincaré section. The 5T-periodic response becomes the 


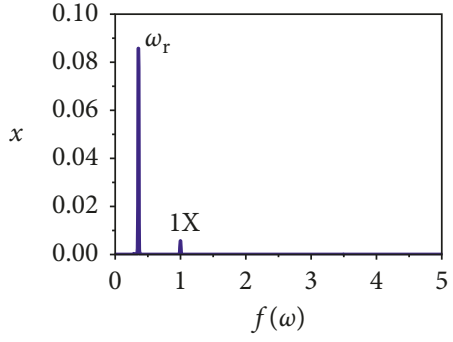

(a)

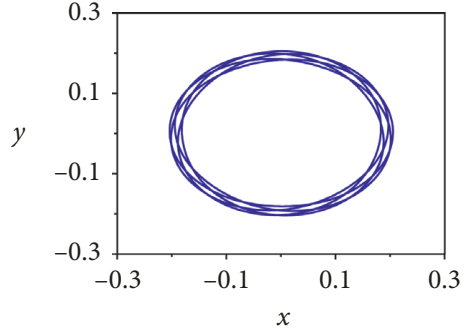

(b)

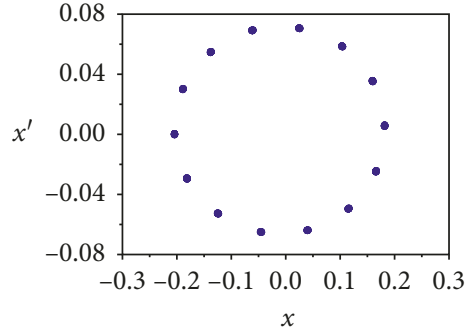

(c)

Figure 8: The 14T-periodic response of the rotor/seal system at $s=2.8$. (a) The frequency spectrum of the $x$ coordinate. (b) The orbit of the rotor. (c) The Poincaré map of the $x$ coordinate.

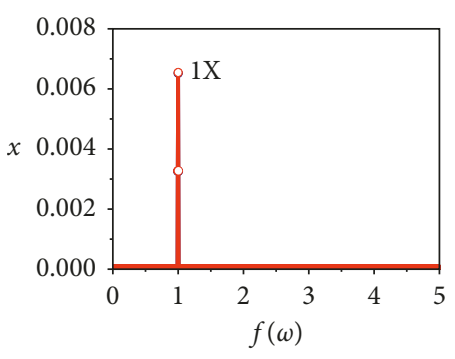

(a)

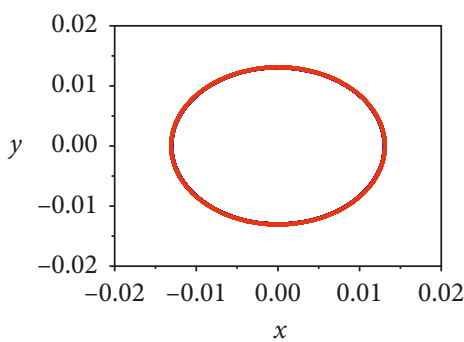

(b)

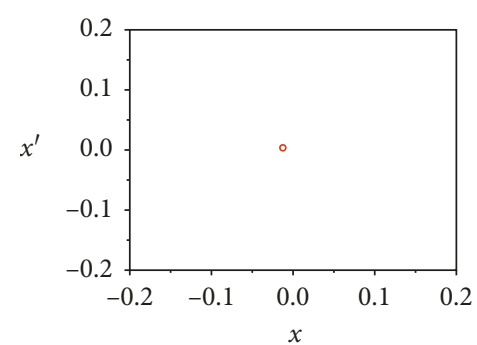

(c)

FIgURE 9: The response of the rotor/seal system with the DVA at $s=2$. (a) The frequency spectrum of the $x$ coordinate. (b) The orbit of the rotor. (c) The Poincaré map of the $x$ coordinate.

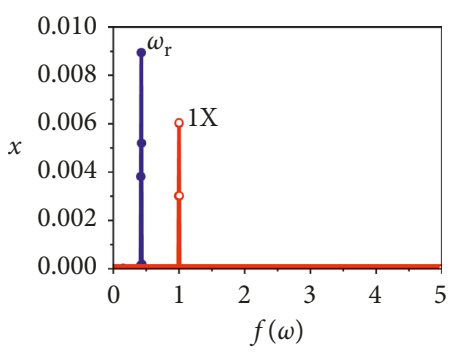

(a)

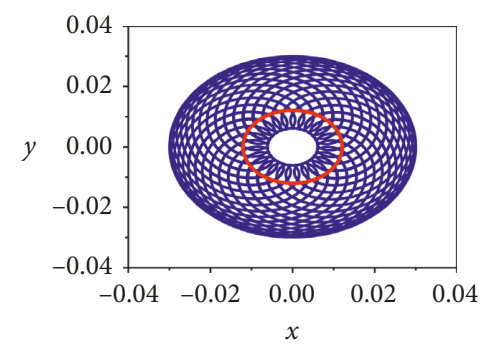

(b)

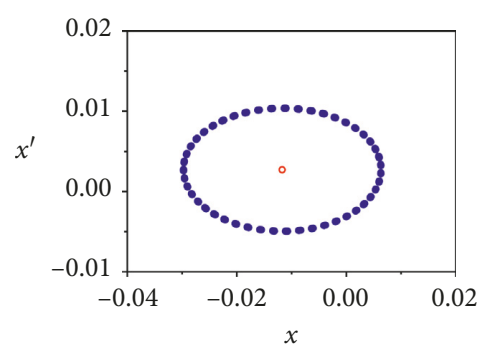

(c)

FIgURE 10: The response of the rotor/seal system with the DVA at $s=2.35$. (a) The frequency spectrum of the $x$ coordinate. (b) The orbit of the rotor. (c) The Poincare map of the $x$ coordinate.

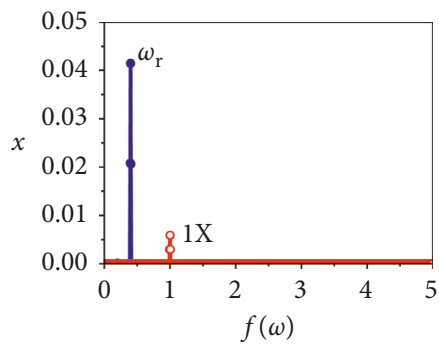

(a)

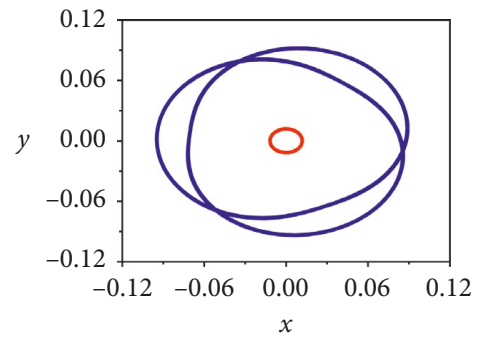

(b)

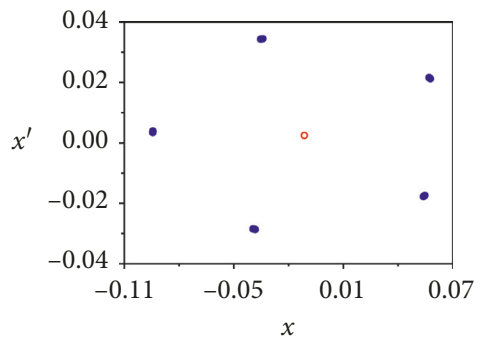

(c)

FIGURE 11: The response of the rotor/seal system with the DVA at $s=2.5$. (a) The frequency spectrum of the $x$ coordinate. (b) The orbit of the rotor. (c) The Poincaré map of the $x$ coordinate. 


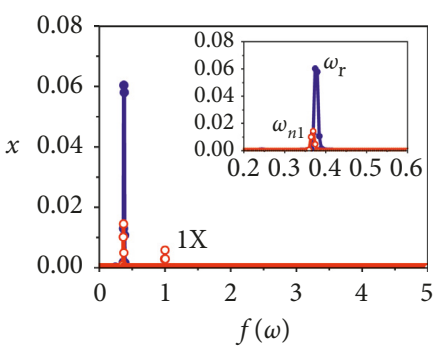

(a)

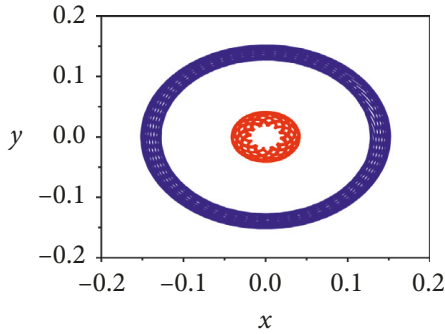

(b)

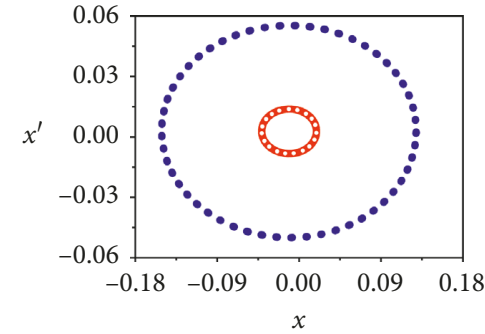

(c)

Figure 12: The response of the rotor/seal system with the DVA at $s=2.65$. (a) The frequency spectrum of the $x$ coordinate. (b) The orbit of the rotor. (c) The Poincaré map of the $x$ coordinate.

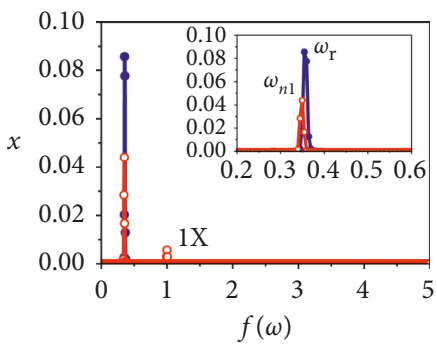

(a)

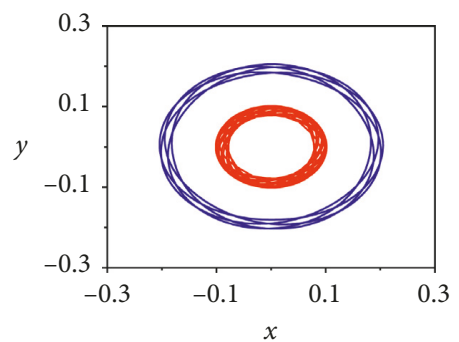

(b)

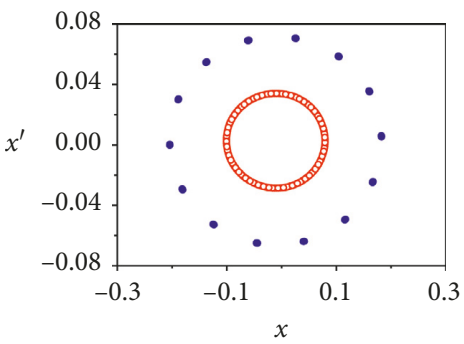

(c)

FIGURE 13: The response of the rotor/seal system with the DVA at $s=2.8$. (a) The frequency spectrum of the $x$ coordinate. (b) The orbit of the rotor. (c) The Poincaré map of the $x$ coordinate.

periodic response. Figure 12 shows that the $1 \mathrm{X}$ frequency and the first-order natural frequency $\omega_{n 1}$ of the rotor-DVA system components exist in the frequency spectrum; that is, the instability vibration frequency changes from the rotor natural frequency $\omega_{\mathrm{r}}$ to the rotor-DVA first-order natural frequency $\omega_{n 1}$. Note that the instability vibration is not eliminated completely, but the amplitude of the instability vibration frequency becomes smaller by the DVA. The $1 \mathrm{X}$ frequency has no change in the frequency spectrum, the orbit of the rotor is also irregular, and a closed curve formed by the infinite points is also in the Poincaré section. The vibration is still quasiperiodic. Figure 13 shows that the $1 \mathrm{X}$ frequency and the first-order natural frequency $\omega_{n 1}$ of the rotor-DVA system components exist in the frequency spectrum; that is, the instability vibration frequency also changes from the rotor natural frequency $\omega_{\mathrm{r}}$ to the rotorDVA first-order natural frequency $\omega_{n 1}$. Note that the instability vibration is not eliminated completely, but the amplitude of the instability vibration frequency also becomes smaller by the DVA. The $1 \mathrm{X}$ frequency has no change in the frequency spectrum, and the orbit of the rotor is also irregular, but a closed curve formed by the infinite points instead of fourteen isolated points is in the Poincaré section. The $14 \mathrm{~T}$ periodic response becomes the quasiperiodic response.

The results from Figures 9-13 show the following: (1) the response away from the resonance region is not affected by the DVA in the synchronous vibration region; (2) the instability vibration frequency can be eliminated completely by the DVA within a certain rotating speed range in the instability vibration region; (3) the instability vibration frequency can be eliminated partially by the DVA within the other rotating speed range in the instability vibration region; (4) the $1 \mathrm{X}$ frequency vibration is not affected by the DVA. The bifurcation diagram and the amplitude-frequency response curve of the rotor/seal system attached with the DVA are established, and the results are shown in Figure 14. The blue solid dot and line are the bifurcation and amplitude-frequency response curve of the rotor/seal system, and the red solid dot and line are that of the rotor/seal system with the DVA, respectively. After adding the DVA, it can be observed in Figure 14(a) that the vibration response is periodic at $s<2.58$. Only one point is correspondingly shown in the bifurcation diagram for every rotating speed. The vibration response is $n \mathrm{~T}$ periodic or quasiperiodic at $s \geq 2.58$ and can be determined by the Poincare map. $s=2.58$ is the instability threshold of the rotor/seal-DVA system.

Compared with the rotor/seal system without the DVA, the instability threshold of the rotor/seal system with the DVA increases by about $12 \%$. The region of the synchronous response is extended, and the occurrence of the fluidinduced vibration is postponed by the DVA. The natural frequency vibration due to the fluid-induced instability in the rotor/seal system can be eliminated completely using the DVA within the rotating speed range between $s=2.31$ and $s$ $=2.58$. This is the rotating speed range of the complete elimination of the instability vibration.

Figure 14(b) shows that the amplitude of the rotor/seal system increases rapidly as the rotating speed exceeds the 


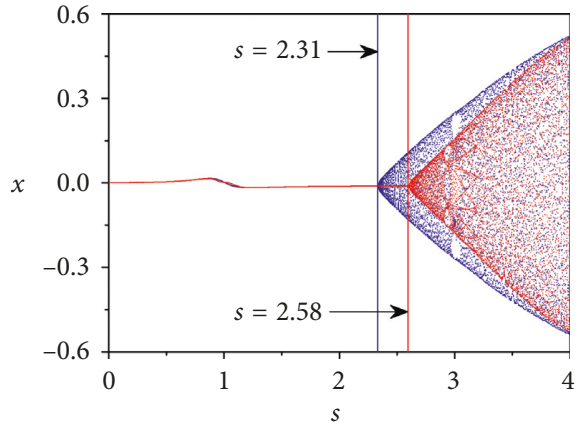

(a)

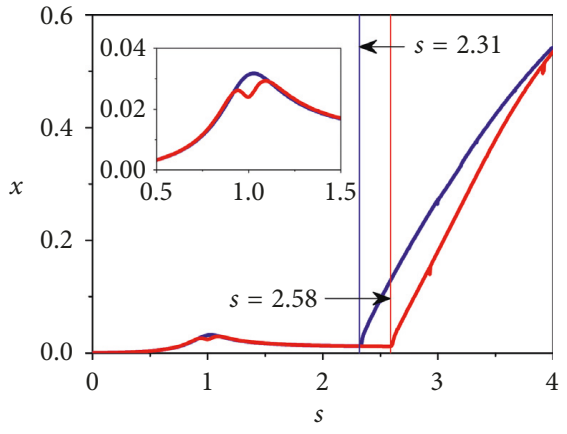

(b)

Figure 14: (a) The bifurcation diagram and (b) the amplitude-frequency response curve of the rotor/seal system attached with the DVA in the $x$ coordinate.

instability threshold of the rotor/seal system with the DVA $s$ $=2.58$ when the DVA is added. Compared with the rotor/seal system without the DVA, the amplitude of the rotor/seal system attached with the DVA is smaller than that of the rotor/seal system as the rotating speed exceeds $s=2.31$. The natural frequency vibration due to the fluid-induced instability in the rotor/seal system can be eliminated partially using the DVA within the rotating speed range $s>2.58$. This is the rotating speed range of the partial elimination of the instability vibration. The amplitudes of the rotor/seal system without and with the DVA are closer as the rotating speed increases.

For the 1X frequency (unbalance) vibration attenuation of the rotor system, the partial enlarged view in Figure 14(b) shows that the amplitude of the rotor can be reduced by the DVA in the resonance region during the vibration process. However, the DVA is ineffective when the $1 \mathrm{X}$ frequency (rotating speed) is away from the resonance region. This is one of the vibration attenuation characteristics of the DVA. It is the reason that the $1 \mathrm{X}$ frequency has almost no change in the frequency spectrum in Figures 9-13.

Compared with the NES, first, the structure of this DVA is easy to realize. The structure of the NES which has an essentially nonlinear stiffness is more complex. Second, the NES is effective when the amplitude of the main system is within a certain range; that is, the NES is ineffective when the amplitude of the main system is too large. This DVA has no such restriction. For the vibration attenuation of the unbalance rotor system in the resonance region, it has been compared between the DVA and NES in Reference [20]. Both DVA and NES can reduce the amplitude of the rotor system in the resonance region, but the system with the DVA has two large amplitude areas in the resonance region, while the system with the NES only has one large amplitude area which is in the resonance region. In a very large frequency range, the system with the NES has lower vibration amplitude than the system with the DVA. However, near the resonance point, the system with the DVA has lower vibration amplitude than the system with the NES. For the fluid-induced vibration attenuation of the rotor/seal system, the effect of this DVA has been discussed in this paper, but the effect of the NES has not been investigated. It is a very interesting work and will be discussed in the future.

The process of the simulation analysis is as follows. First, the model of the rotor/seal system is established. The modified Jeffcott rotor and Muszynska nonlinear seal force models are applied for the simulation analysis. Then, the numerical method is used due to the nonlinear term. The dynamic characteristics of the rotor/seal system are analysed with the bifurcation diagrams, amplitudefrequency response curves, frequency spectrum, trajectory diagrams, and Poincaré maps. Third, the DVA is attached in the rotor/seal system. The motion equation of the rotor/seal-DVA system is established. The initial parameters of the DVA are selected. The numerical method is also used for the nonlinear characteristic analysis. Finally, the dynamic characteristics of the rotor/seal system without and with the DVA are compared. The effect of the DVA on the instability vibration elimination is obtained.

\section{Influences of the Parameters of the DVA on the Instability Threshold}

The instability threshold is a key parameter for the rotor instability vibration elimination from the above analysis. The rotating speed ranges of the complete and partial elimination of the instability vibration are determined by the instability threshold. So, the effects of the natural frequency and damping ratio of the DVA on the instability threshold are investigated in this section.

4.1. Influence of the Natural Frequency of the DVA on the Instability Threshold. The effect of the natural frequency of the DVA on the instability threshold for the rotor/seal system with the DVA is considered first. Assuming the nondimensional natural frequency of the DVA $\sigma_{\mathrm{a}}=\omega_{\mathrm{a}} / \omega_{\mathrm{r}}$. Figure 15 represents that the instability threshold of the rotor/seal system changes with the natural frequency of the DVA as $\zeta_{\mathrm{a}}=0.05$. The blue solid line is the instability threshold of the rotor/seal system, and the red solid line is the instability threshold of the rotor/seal system with the 


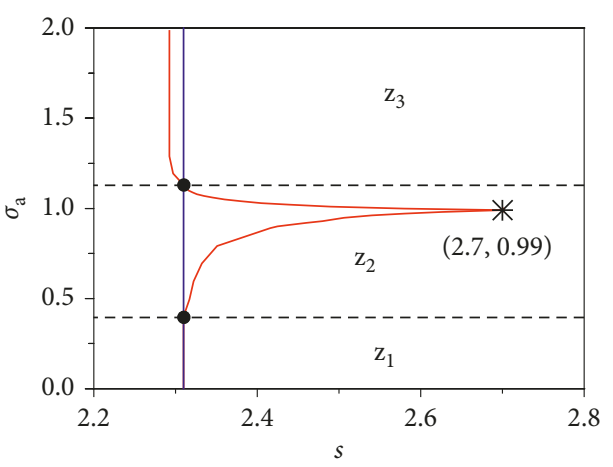

Figure 15: The effect of the natural frequency of the DVA on the instability threshold of the rotor/seal system as $\zeta_{\mathrm{a}}=0.05$.

DVA, respectively. Accordingly, it can be seen that the instability thresholds of the rotor/seal system with and without the DVA are equal in the zone of $z_{1}$, the instability threshold of the rotor system with the DVA is greater than that of the system without the DVA in the zone of $z_{2}$, and the instability threshold of the rotor/seal system with the DVA is less than that of the system without the DVA in the zone of $z_{3}$. Consequently, the DVA natural frequency ranges by which the instability vibration can be eliminated completely and partially is in the zone of $\mathrm{z}_{2}$ (about $0.4<\sigma_{\mathrm{a}}<1.1$ ). The region consisting of the blue and red solid lines is the complete elimination of the instability vibration. The region consisting of the red solid line and two black dash lines is the partial elimination of the instability vibration. The instability threshold of the rotor/seal system attached with the DVA increases first and then decreases as $\sigma_{\mathrm{a}}$ increases, and the maximum is $s=2.7$ when $\sigma_{\mathrm{a}}$ is about 0.99 . So, the rotating speed range of the complete elimination of the instability vibration can be extended. The natural frequency vibration due to the fluid-induced instability in the rotor/seal system can be eliminated completely using the DVA within the maximum rotating speed range between $s=2.31$ and $s=2.7$.

Case 1. In the maximum case of picking $\sigma_{\mathrm{a}}=0.99$ and $\zeta_{\mathrm{a}}=$ 0.05 when the natural frequency of the DVA changes in Figure 15, the bifurcation diagram and the amplitudefrequency response curve of the rotor/seal system attached with the DVA are established, and the results are shown in Figure 16. Compared with the rotor/seal system without the DVA, the instability threshold of the rotor/seal system with the DVA increases by about $17 \%$.

4.2. Influence of the Damping Ratio of the DVA on the Instability Threshold. The effect of the damping ratio of the DVA on the instability threshold for the rotor/seal system with the DVA is considered as follows. Figure 17 represents that the instability threshold of the rotor/seal system changes with the damping ratio of the DVA as $\sigma_{\mathrm{a}}=1$. The blue and red solid lines are the instability threshold of the rotor/seal system without and with the DVA, respectively. It shows that the instability threshold of the rotor/seal system with the DVA is less than that of the system without the DVA in the zone of $z_{1}$, and the instability threshold of the rotor system

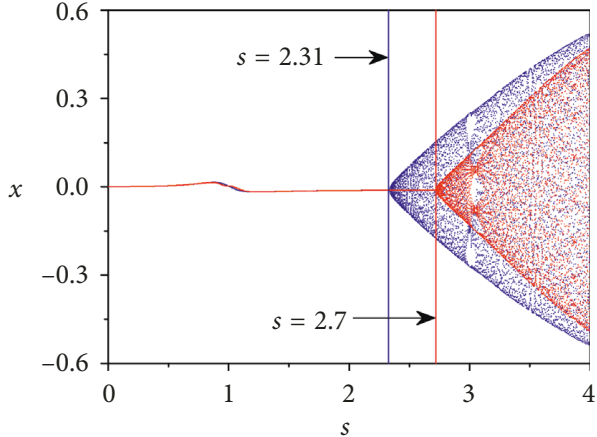

(a)

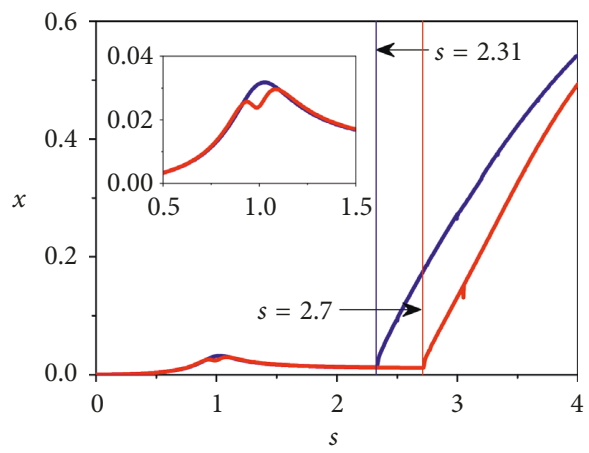

(b)

FIgURE 16: (a) The bifurcation diagram and (b) the amplitudefrequency response curve of the rotor/seal system attached with the DVA in the $x$ coordinate when $\zeta_{\mathrm{a}}=0.05$ and $\sigma_{\mathrm{a}}=0.99$.

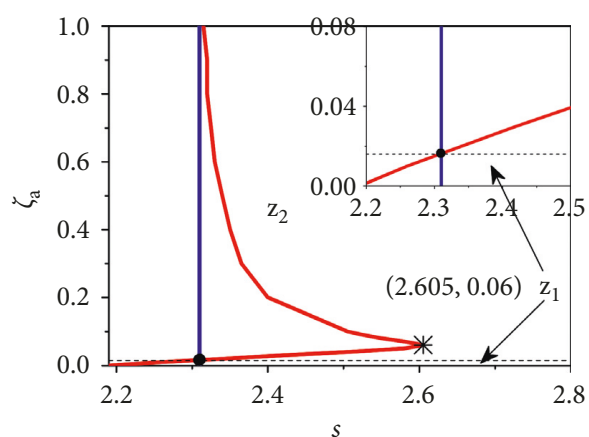

FIgUre 17: The effect of the damping ratio of the DVA on the instability threshold of the rotor/seal system as $\sigma_{\mathrm{a}}=1$.

with the DVA is greater than that of the system without the DVA in the zone of $z_{2}$. Consequently, the DVA damping ratio range by which the instability vibration can be eliminated completely and partially is in the zone of $z_{2}$ (about $\left.0.02<\zeta_{\mathrm{a}}<1\right)$. The region consisting of the blue and red solid lines is the complete elimination of the instability vibration. The region consisting of the red solid line and the black dash line is the partial elimination of the instability vibration. The instability threshold of the rotor/seal system with the DVA increases first and then decreases as $\zeta_{\mathrm{a}}$ increases, the maximum is $s=2.605$ when $\zeta_{\mathrm{a}}$ is about 0.06 . So, the rotating speed range of the complete elimination of the instability vibration can be extended. The natural frequency vibration 


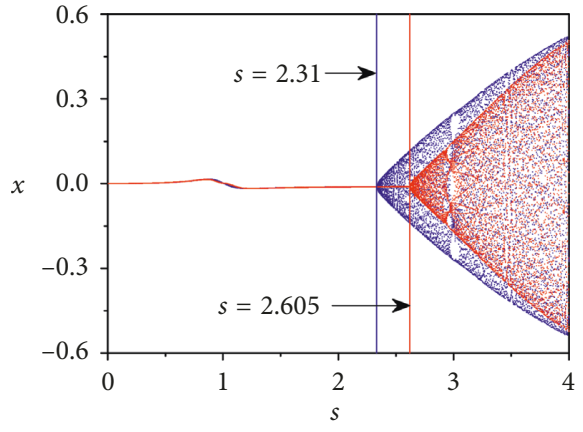

(a)

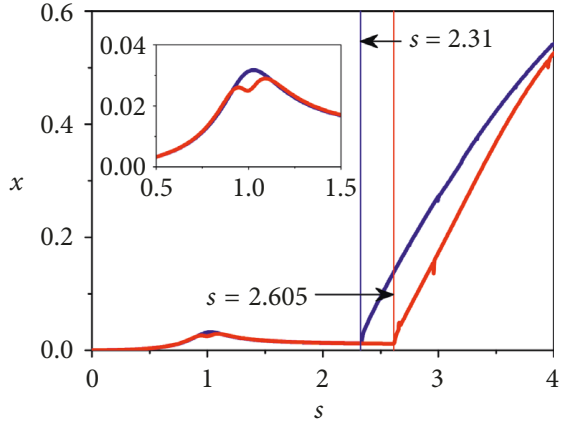

(b)

FiguRE 18: (a) The bifurcation diagram and (b) the amplitude-frequency response curve of the rotor/seal system attached with the DVA in the $x$ coordinate when $\zeta_{\mathrm{a}}=0.06$ and $\sigma_{\mathrm{a}}=1$.

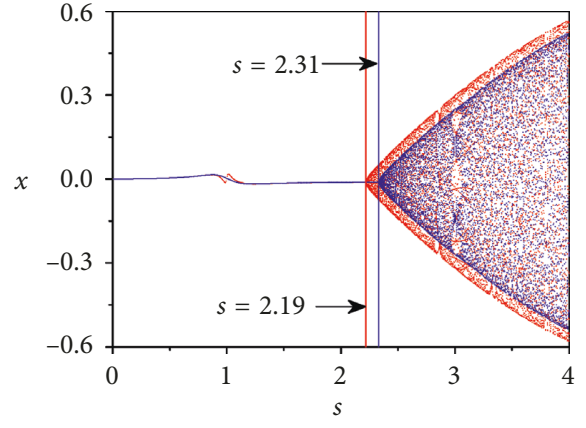

(a)

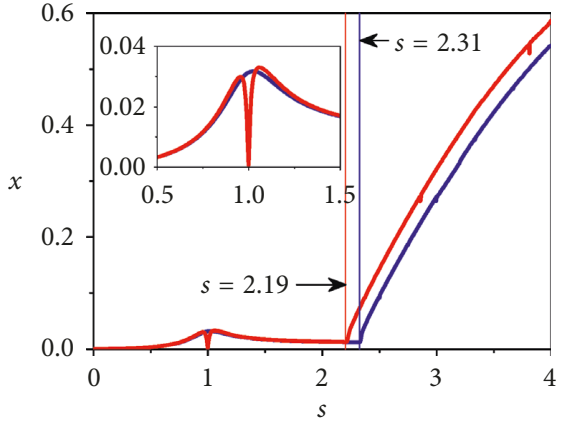

(b)

Figure 19: (a) The bifurcation diagram and (b) the amplitude-frequency response curve of the rotor/seal system attached with the DVA in the $x$-coordinate when $\zeta_{\mathrm{a}}=0$ and $\sigma_{\mathrm{a}}=1$.

due to the fluid-induced instability in the rotor/seal system can be eliminated completely using the DVA within the maximum rotating speed range between $s=2.31$ and $s=$ 2.605 .

Case 2. In the maximum case of picking $\zeta_{\mathrm{a}}=0.06$ and $\sigma_{\mathrm{a}}=1$ when the damping ratio of the DVA changes in Figure 17, the bifurcation diagram and the amplitude-frequency response curve of the rotor/seal system attached with the DVA are established and the results are shown in Figure 18. Compared with the rotor/seal system without the DVA, the instability threshold of the rotor/seal system with the DVA increases by about $13 \%$.

Case 3. Especially, picking $\zeta_{\mathrm{a}}=0$ and $\sigma_{\mathrm{a}}=1$ in Figure 17, the absorber is the undamped DVA. The bifurcation diagram and the amplitude-frequency response curve of the rotor/seal system attached with the undamped DVA are established and the results are shown in Figure 19. The blue solid dot and line are the bifurcation and amplitudefrequency response curve of the rotor/seal system, and the red solid dot and line are that of the rotor/seal system with the DVA, respectively. After adding the DVA, it can be observed in Figure 19(a) that the instability threshold of the rotor/seal system with the DVA $(s=2.19)$ is less than that of the system without the DVA $(s=2.31)$; that is, the instability vibration occurs in advance when the undamped DVA is mounted on the rotor/seal system. Figure 19(b) shows that the amplitude of the rotor/seal system increases rapidly as the rotating speed exceeds $s=2.19$ when the undamped DVA is added. Compared with the rotor/seal system without the DVA, the amplitude of the rotor/seal system attached with the DVA is greater than that of the rotor/seal system as the rotating speed exceeds $s=2.19$.

The results from Figure 19 show that the natural frequency vibration due to the fluid-induced instability in the rotor/seal system cannot be eliminated using the undamped DVA. The instability threshold decreases by about $5 \%$.

Case 4. Especially, picking $\zeta_{\mathrm{a}}=1$ and $\sigma_{\mathrm{a}}=1$ in Figure 17, the absorber is the DVA with critical damping. The bifurcation diagram and the amplitude-frequency response curve of the rotor/seal system attached with the DVA are established, and the results are shown in Figure 20. After adding the DVA, it can be observed that the instability threshold and the amplitude of the instability vibration of the rotor/seal system with the DVA are very close to those of the rotor/seal system without the DVA. There is almost no effect on the instability threshold and the amplitude of the instability vibration of the rotor/seal system after the DVA is added.

The instability thresholds of the rotor/seal system under the above four parameter combinations of the DVA are 


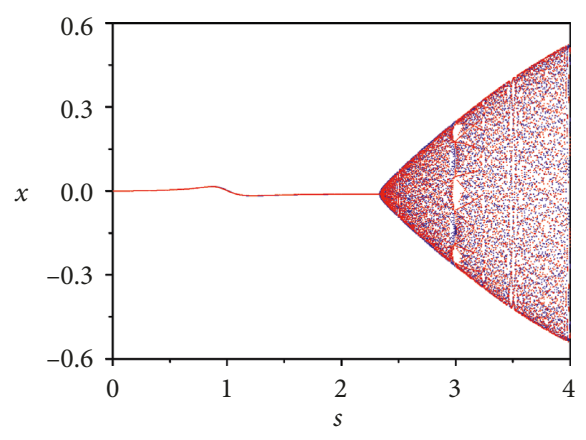

(a)

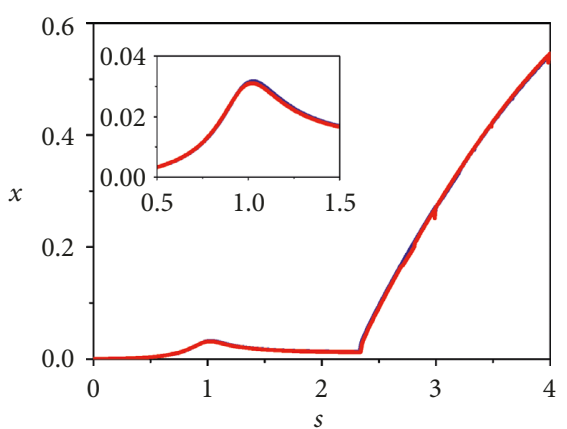

(b)

Figure 20: (a) The bifurcation diagram and (b) the amplitude-frequency response curve of the rotor/seal system attached with the DVA in the $x$ coordinate when $\zeta_{\mathrm{a}}=1$ and $\sigma_{\mathrm{a}}=1$.

listed as shown in Table 1 . The instability threshold is increased in Cases 1 and 2. Increment of the instability threshold in Case 1 is greater than that in Case 2. The instability threshold is decreased in Case 3 and almost constant in Case 4.

\section{Conclusions}

The fluid-induced vibration elimination in the rotor/seal system attached with the DVA is proposed in this paper. The simplified model of the rotor/seal system with the DVA is established. The Muszynska fluid force model is used for the nonlinear seal force. The Newmark time integration method is adopted to investigate the nonlinear dynamic responses of the rotor/seal system without and with the DVA. The rotating speed ranges by which the fluid-induced instability can be eliminated completely and partially are obtained, respectively. The effects of the natural frequency and damping ratio of the DVA on the fluid-induced vibration elimination are investigated and the DVA parameter ranges by which the instability vibration can be eliminated completely and partially are obtained. The conclusions are drawn as follows:

(1) The proposed method is effective to eliminate the natural frequency vibration due to the fluid-induced instability of the rotor/seal system. For the certain rotating speed range, the instability vibration can be eliminated completely. The $1 \mathrm{X}$ frequency vibration is not affected by the DVA since the rotating speed is away from the resonance region.

(2) The amplitude of the instability vibration in the rotor/seal system can be decreased using the DVA within the rotating speed range of the partial elimination of the instability vibration.

(3) The natural frequency and the damping ratio of the DVA have a greater impact on the instability vibration elimination effect when the mass of the DVA is constant. The DVA has a higher efficiency when picking $\left(\sigma_{\mathrm{a}}=0.99, \zeta_{\mathrm{a}}=0.05\right)$ or $\left(\sigma_{\mathrm{a}}=1, \zeta_{\mathrm{a}}=0.06\right)$. Especially, the instability vibration occurs in advance when picking $\left(\sigma_{\mathrm{a}}=1, \zeta_{\mathrm{a}}=0\right)$, and there is almost no
TABLE 1: The instability thresholds of the rotor/seal system under the four parameter combinations of the DVA.

\begin{tabular}{lcccc}
\hline Case & $\sigma_{\mathrm{a}}$ & $\zeta_{\mathrm{a}}$ & $\begin{array}{c}\text { Instability } \\
\text { threshold }\end{array}$ & $\begin{array}{c}\text { Increment of instability } \\
\text { threshold }\end{array}$ \\
\hline Rotor without & - & - & 2.31 & - \\
DVA & - & - & & $+17 \%$ \\
1 & 0.99 & 0.05 & 2.7 & $+13 \%$ \\
2 & 1 & 0.06 & 2.605 & $-5 \%$ \\
3 & 1 & 0 & 2.19 & 0 \\
4 & 1 & 1 & 2.31 & \\
\hline
\end{tabular}

effect on the instability vibration elimination when picking $\left(\sigma_{\mathrm{a}}=1, \zeta_{\mathrm{a}}=1\right)$.

Further works include the comparison between simulation and experiment for the effectiveness of the DVA on the rotor test rig.

\section{Appendix}

The Newmark time integration method:

The differential equation of the rotor system is given by

$$
\mathbf{M} \ddot{\mathbf{x}}+\mathbf{C} \dot{\mathbf{x}}+\mathbf{K x}+\mathbf{f}(\mathbf{x})=\mathbf{P},
$$

where $\mathbf{f}(\mathbf{x})$ is the nonlinear restoring force.

The equation at time $t$ is given by

$$
\mathbf{M} \ddot{\mathbf{x}}^{t}+\mathbf{C} \dot{\mathbf{x}}^{t}+\mathbf{K} \mathbf{x}^{t}+\mathbf{f}\left(\mathbf{x}^{t}\right)=\mathbf{P}^{t} .
$$

The equation at time $t+\Delta t$ is given by

$$
\mathbf{M} \ddot{\mathbf{x}}^{t+\Delta t}+\mathbf{C} \dot{\mathbf{x}}^{t+\Delta t}+\mathbf{K} \mathbf{x}^{t+\Delta t}+\mathbf{f}\left(\mathbf{x}^{t+\Delta t}\right)=\mathbf{P}^{t+\Delta t} .
$$

If $\mathbf{f}(\mathbf{x})$ is differentiable in the neighbourhood of $\mathbf{x}$, $\mathbf{f}\left(\mathbf{x}^{t+\Delta t}\right)$ can be expanded with respect to $\mathbf{x}^{t}$ by the Taylor series:

$$
\mathbf{f}\left(\mathbf{x}^{t+\Delta t}\right)=\mathbf{f}\left(\mathbf{x}^{t}\right)+\left.\frac{\partial \mathbf{f}}{\partial \mathbf{x}}\right|_{x=x^{t}} \Delta \mathbf{x}+\frac{1}{2 !}\left(\left.\frac{\partial^{2} \mathbf{f}}{\partial \mathbf{x}^{2}}\right|_{x=x^{t}}\right) \Delta \mathbf{x}^{2}+\cdots
$$

where $\Delta \mathbf{x}=\mathbf{x}^{t+\Delta t}-\mathbf{x}^{t}$ are the incremental displacement. 
The higher order terms beyond the second derivatives are neglected; then, Equation (11) can be reduced to

$$
\mathbf{f}\left(\mathbf{x}^{t+\Delta t}\right)=\mathbf{f}\left(\mathbf{x}^{t}\right)+\mathbf{K}_{\mathrm{T}}^{t} \Delta \mathbf{x}
$$

where

$$
\mathbf{K}_{\mathrm{T}}^{t}=\left.\frac{\partial \mathbf{f}}{\partial \mathbf{x}}\right|_{x=x^{t}}
$$

Substituting Equation (12) into Equation (10), the original equation becomes an incremental equation:

$$
\mathbf{M} \ddot{\mathbf{x}}^{t+\Delta t}+\mathbf{C} \dot{\mathbf{x}}^{t+\Delta t}+\mathbf{K} \mathbf{x}^{t+\Delta t}+\mathbf{K}_{\mathrm{T}}^{t} \Delta \mathbf{x}=\mathbf{P}^{t+\Delta t}-\mathbf{f}\left(\mathbf{x}^{t}\right) .
$$

Assume that

$$
\left\{\begin{array}{l}
\dot{\mathbf{x}}^{t+\Delta t}=\dot{\mathbf{x}}^{t}+\left[(1-\delta) \ddot{\mathbf{x}}^{t}+\delta \ddot{\mathbf{x}}^{t+\Delta t}\right] \Delta t \\
\mathbf{x}^{t+\Delta t}=\mathbf{x}^{t}+\dot{\mathbf{x}}^{t} \Delta t+\left[\left(\frac{1}{2}-\beta\right) \ddot{\mathbf{x}}^{t}+\beta \ddot{\mathbf{x}}^{t+\Delta t}\right] \Delta t^{2},
\end{array}\right.
$$

where $\delta \geq 0.5$ and $\beta \geq 0.25(0.5+\delta)^{2}$. Then,

$$
\left\{\begin{array}{l}
\ddot{\mathbf{x}}^{t+\Delta t}=\frac{1}{\beta \Delta t^{2}}\left(\mathbf{x}^{t+\Delta t}-\mathbf{x}^{t}\right)-\frac{1}{\beta \Delta t} \dot{\mathbf{x}}^{t}-\left(\frac{1}{2 \beta}-1\right) \ddot{\mathbf{x}}^{t}, \\
\dot{\mathbf{x}}^{t+\Delta t}=\frac{\delta}{\beta \Delta t}\left(\mathbf{x}^{t+\Delta t}-\mathbf{x}^{t}\right)-\left(\frac{\delta}{\beta}-1\right) \dot{\mathbf{x}}^{t}-\left(\frac{\delta}{2 \beta}-1\right) \Delta t \ddot{\mathbf{x}}^{t} .
\end{array}\right.
$$

Assume that

$$
\begin{aligned}
& a_{0}=\frac{1}{\beta \Delta t^{2}}, \\
& a_{1}=\frac{\delta}{\beta \Delta t}, \\
& a_{2}=\frac{1}{\beta \Delta t}, \\
& a_{3}=\frac{1}{2 \beta}-1, \\
& a_{4}=\frac{\delta}{\beta}-1, \\
& a_{5}=\left(\frac{\delta}{2 \beta}-1\right) \Delta t, \\
& a_{6}=(1-\delta) \Delta t, \\
& a_{7}=\delta \Delta t .
\end{aligned}
$$

Substituting Equations (17) and (18) into Equation (16), Equation (16) is rewritten as

$$
\left\{\begin{array}{l}
\ddot{\mathbf{x}}^{t+\Delta t}=a_{0} \Delta \mathbf{x}-a_{2} \dot{\mathbf{x}}^{t}-a_{3} \ddot{\mathbf{x}}^{t} \\
\dot{\mathbf{x}}^{t+\Delta t}=a_{1} \Delta \mathbf{x}-a_{4} \dot{\mathbf{x}}^{t}-a_{5} \ddot{\mathbf{x}}^{t}=\dot{\mathbf{x}}^{t}+a_{6} \ddot{\mathbf{x}}^{t}+a_{7} \ddot{\mathbf{x}}^{t+\Delta t} .
\end{array}\right.
$$

$\Delta \dot{\mathbf{x}}$ and $\Delta \ddot{\mathbf{x}}$ are written as

$$
\left\{\begin{array}{l}
\Delta \dot{\mathbf{x}}=\dot{\mathbf{x}}^{t+\Delta t}-\dot{\mathbf{x}}^{t}=a_{1} \Delta \mathbf{x}-\left(a_{4}+1\right) \dot{\mathbf{x}}^{t}-a_{5} \ddot{\mathbf{x}}^{t}, \\
\Delta \ddot{\mathbf{x}}=\ddot{\mathbf{x}}^{t+\Delta t}-\ddot{\mathbf{x}}^{t}=a_{0} \Delta \mathbf{x}-a_{2} \dot{\mathbf{x}}^{t}-\left(a_{3}+1\right) \ddot{\mathbf{x}}^{t} .
\end{array}\right.
$$

Substituting Equations (19) and (20) into the incremental equation, Equation (14) is rewritten as

$$
\begin{aligned}
{\left[a_{0} \mathbf{M}+a_{1} \mathbf{C}+\left(\mathbf{K}+\mathbf{K}_{\mathrm{T}}^{t}\right)\right] \Delta \mathbf{x}=} & \mathbf{P}^{t+\Delta t}-\mathbf{f}\left(\mathbf{x}^{t}\right)-\mathbf{K} \mathbf{x}^{t} \\
& +\mathbf{M}\left(a_{2} \dot{\mathbf{x}}^{t}+a_{3} \ddot{\mathbf{x}}^{t}\right) \\
& +\mathbf{C}\left(a_{4} \dot{\mathbf{x}}^{t}+a_{5} \ddot{\mathbf{x}}^{t}\right)
\end{aligned}
$$

Assume that

$$
\left\{\begin{array}{l}
\widehat{K}_{\mathrm{T}}^{t}=a_{0} \mathbf{M}+a_{1} \mathbf{C}+\left(\mathbf{K}+\mathbf{K}_{\mathrm{T}}^{t}\right), \\
\widehat{P}^{t+\Delta t}=\mathbf{P}^{t+\Delta t}-\mathbf{f}\left(\mathbf{x}^{t}\right)-\mathbf{K} \mathbf{x}^{t}+\mathbf{M}\left(a_{2} \dot{\mathbf{x}}^{t}+a_{3} \ddot{\mathbf{x}}^{t}\right)+\mathbf{C}\left(a_{4} \dot{\mathbf{x}}^{t}+a_{5} \ddot{\mathbf{x}}^{t}\right) .
\end{array}\right.
$$

Substituting Equation (22) into Equation (21), Equation (21) is rewritten as

$$
\widehat{K}_{\mathrm{T}}^{t} \Delta \mathbf{x}=\widehat{P}^{t+\Delta t}
$$

The converge criteria is defined by

$$
\frac{\left\|{ }^{(i)} \delta \mathbf{x}\right\|}{\left\|{ }^{(i)} \Delta \mathbf{x}^{t+\Delta t}\right\|},
$$

where $\|\cdot\|$ is the Euclidean norm. The iteration repeats until the above criterion is less than a tolerance. The brief procedures of the iteration scheme are stated as follows:

Initialize $\mathbf{x}, \dot{\mathbf{x}}$, and $\ddot{\mathbf{x}}$ and solve for the displacement increment $\Delta \mathbf{x}$ by Equation (23).

(1) $i=i+1$

Calculate the $(i-1)^{\text {th }}$ approximation $\ddot{\mathbf{x}}, \dot{\mathbf{x}}$, and $\mathbf{x}$

$$
\left\{\begin{aligned}
{ }^{(i-1)} \ddot{\mathbf{x}} t+\Delta t & =a_{0}^{(i-1)} \Delta \mathbf{x}-a_{2} \dot{\mathbf{x}}^{t}-a_{3} \ddot{\mathbf{x}}^{t}, \\
{ }^{(i-1)} \dot{\mathbf{x}} t+\Delta t & =a_{1}^{(i-1)} \Delta \mathbf{x}-a_{4} \dot{\mathbf{x}}^{t}-a_{5} \ddot{\mathbf{x}}^{t} \\
{ }^{(i-1)} \mathbf{x} t+\Delta t & ={ }^{(i-1)} \Delta \mathbf{x}+\mathbf{x}^{t} .
\end{aligned}\right.
$$

(2) Calculate the ${ }^{(i-1)} \widehat{K} \mathrm{~T}_{t}$ and ${ }^{(i-1)} \mathbf{f}\left(\mathbf{x}^{t+\Delta t}\right)$

$$
\left\{\begin{array}{l}
{ }^{(i-1)} \widehat{K} \mathrm{~T}_{t}=a_{0} \mathbf{M}+a_{1} \mathbf{C}+\left(\mathbf{K}+{ }^{(i-1)} \mathbf{K T}_{t}\right) \\
{ }^{(i-1)} \mathbf{f}\left(\mathbf{x}^{t+\Delta t}\right)=\mathbf{f}\left({ }^{(i-1)} \mathbf{x} t+\Delta t\right)
\end{array}\right.
$$

(3) Calculate the ${ }^{(i-1)} \widehat{P} t+\Delta t$

$$
\begin{gathered}
{ }^{(i-1) \widehat{P}} t+\Delta t=\mathbf{P}^{t+\Delta t}-\mathbf{M}^{(i-1)} \ddot{\mathbf{x}} t+\Delta t-\mathbf{C}^{(i-1)} \dot{\mathbf{x}} t \\
+\Delta t-\mathbf{K}^{(i-1)} \mathbf{x} t+\Delta t-{ }^{(i-1)} \mathbf{f}\left(\mathbf{x}^{t+\Delta t}\right) .
\end{gathered}
$$


(4) Calculate the displacement increment $\delta \mathbf{x}$

$$
{ }^{(i-1)} \widehat{K} \mathrm{~T}_{t} \delta \mathbf{x}={ }^{(i-1)} \widehat{P} t+\Delta t
$$

(5) Calculate the new displacement increment $\Delta \mathbf{x}$

$$
{ }^{(i)} \Delta \mathbf{x}={ }^{(i-1)} \Delta \mathbf{x}+\delta \mathbf{x} .
$$

(6) Check the convergence criteria. If no convergence, go to step 1 , if convergence, then the $\ddot{\mathbf{x}}, \dot{\mathbf{x}}$, and $\mathbf{x}$ at time $t+\Delta t$ are given by

$$
\left\{\begin{array}{l}
\ddot{\mathbf{x}}^{t+\Delta t}=a_{0} \Delta \mathbf{x}-a_{2} \dot{\mathbf{x}}^{t}-a_{3} \ddot{\mathbf{x}}^{t} \\
\dot{\mathbf{x}}^{t+\Delta t}=a_{1} \Delta \mathbf{x}-a_{4} \dot{\mathbf{x}}^{t}-a_{5} \ddot{\mathbf{x}}^{t} \\
\mathbf{x}^{t+\Delta t}=\Delta \mathbf{x}+\mathbf{x}^{t}
\end{array}\right.
$$

\section{Data Availability}

The data used to support the findings of this study are included within the article.

\section{Conflicts of Interest}

The authors declare that there are no conflicts of interest regarding the publication of this paper.

\section{Acknowledgments}

The authors would like to gratefully acknowledge the National Natural Science Foundation of China (grant numbers 51475085 and U1708257) and 2017 Higher Education Institution Basic Research Project of Liaoning Province (grant number LQGD2017019) for the financial support for this study.

\section{References}

[1] N. Bachschmid, P. Pennacchi, and A. Vania, "Steam-whirl analysis in a high pressure cylinder of a turbo generator," Mechanical Systems and Signal Processing, vol. 22, no. 1, pp. 121-132, 2008.

[2] A. Muszynska and D. E. Bently, "Anti-swirl arrangements prevent rotor/seal instability," Journal of Vibration, Acoustics, Stress, and Reliability in Design, vol. 111, no. 2, pp. 156-162, 1989.

[3] D. Le, C. Tsuei, and M. Pan, "Optimally controlled anti-swirl injection to eliminate whirl in fluid-film bearings of rotary machinery," International Journal of Mechanical Sciences, vol. 98, pp. 157-168, 2015.

[4] D. Sun, S. Wang, Y. Ai et al., "Theoretical and experimental research on the performance of anti-swirl flow for the static and dynamic characteristics of seals," Journal of Mechanical Engineering, vol. 52, no. 3, pp. 101-109, 2016.

[5] D. Childs, D. Elrod, and K. Hale, "Annular honeycomb seals: test results for leakage and rotordynamic coefficients; comparisons to labyrinth and smooth configurations," Journal of Tribology, vol. 111, no. 2, pp. 293-301, 1989.
[6] S. Subramanian, A. S. Sekhar, and B. V. S. S. Prasad, "Influence of combined radial location and growth on the leakage performance of a rotating labyrinth gas turbine seal," Journal of Mechanical Science and Technology, vol. 29, no. 6, pp. 2535-2545, 2015.

[7] B. H. Ertas, A. Delgado, and G. Vannini, "Rotordynamic force coefficients for three types of annular gas seals with inlet preswirl and high differential pressure ratio," Journal of Engineering for Gas Turbines and Power-Transactions of the ASME, vol. 134, no. 4, p. 042503, 2012.

[8] A. Chasalevris and F. Dohnal, "Improving stability and operation of turbine rotors using adjustable journal bearings," Tribology International, vol. 104, pp. 369-382, 2016.

[9] J. G. Salazar and I. F. Santos, "Active tilting-pad journal bearings supporting flexible rotors: part I - the hybrid lubrication," Tribology International, vol. 107, pp. 94-105, 2017.

[10] J. G. Salazar and I. F. Santos, "Active tilting-pad journal bearings supporting flexible rotors: part II-the model-based feedback-controlled lubrication," Tribology International, vol. 107, pp. 106-115, 2017.

[11] A. C. Varela and I. F. Santos, "Component level study of an actively lubricated LEG tilting pad bearing: theory and experiment," Tribology International, vol. 120, pp. 115-126, 2018.

[12] M. D. Queiroz, "An active hydrodynamic bearing for controlling self-excited vibrations: theory and simulation," Journal of Vibration and Control, vol. 19, no. 14, pp. 22112222, 2012.

[13] C.-C. Fan and M.-C. Pan, "Active elimination of oil and dry whips in a rotating machine with an electromagnetic actuator," International Journal of Mechanical Sciences, vol. 53, no. 2, pp. 126-134, 2011.

[14] B. Riemann, E. A. Perini, K. L. Cavalca, H. F. D. Castro, and S. Rinderknecht, "Oil whip instability control using $\mu$-synthesis technique on a magnetic actuator," Journal of Sound and Vibration, vol. 332, no. 4, pp. 654-673, 2013.

[15] M. Parseh, M. Dardel, and M. H. Ghasemi, "Performance comparison of nonlinear energy sink and linear tuned mass damper in steady-state dynamics of a linear beam," Nonlinear Dynamics, vol. 81, no. 4, pp. 1981-2002, 2015.

[16] J. E. Chen, W. He, W. Zhang, M. H. Yao, J. Liu, and M. Sun, "Vibration suppression and higher branch responses of beam with parallel nonlinear energy sinks," Nonlinear Dynamics, vol. 91, no. 2, pp. 885-904, 2018.

[17] A. E. Mamaghani, S. E. Khadem, and S. Bab, "Vibration control of a pipe conveying fluid under external periodic excitation using a nonlinear energy sink," Nonlinear Dynamics, vol. 86, no. 3, pp. 1761-1795, 2016.

[18] N. Ebrahimzade, M. Dardel, and R. Shafaghat, "Performance comparison of linear and nonlinear vibration absorbers in aeroelastic characteristics of a wing model," Nonlinear Dynamics, vol. 86, no. 2, pp. 1075-1094, 2016.

[19] H. Yao, Z. Chen, and B. Wen, "Dynamic vibration absorber with negative stiffness for rotor system," Shock and Vibration, vol. 2016, Article ID 5231704, 13 pages, 2016.

[20] H. Yao, D. Zheng, and B. Wen, "Magnetic nonlinear energy sink for vibration attenuation of unbalanced rotor system," Shock and Vibration, vol. 2017, Article ID 4132607, 15 pages, 2017.

[21] H. Hu and L. He, "Online control of critical speed vibrations of a single-span rotor by a rotor dynamic vibration absorber at different installation positions," Journal of Mechanical Science and Technology, vol. 31, no. 5, pp. 2075-2081, 2017. 
[22] S. Bab, S. E. Khadem, and M. Shahgholi, "Lateral vibration attenuation of a rotor under mass eccentricity force using non-linear energy sink," International Journal of Non-Linear Mechanics, vol. 67, pp. 251-266, 2014.

[23] S. Bab, S. E. Khadem, and M. Shahgholi, "Vibration attenuation of a rotor supported by journal bearings with nonlinear suspensions under mass eccentricity force using nonlinear energy sink," Meccanica, vol. 50, no. 9, pp. 2441-2460, 2015.

[24] S. Bab, S. E. Khadem, M. Shahgholi, and A. Abbasi, "Vibration attenuation of a continuous rotor-blisk-journal bearing system employing smooth nonlinear energy sinks," Mechanical Systems and Signal Processing, vol. 84, pp. 128-157, 2017.

[25] C. Guo, M. A. Al-Shudeifat, A. F. Vakakis, L. A. Bergman, D. M. McFarland, and J. Yan, "Vibration reduction in unbalanced hollow rotor systems with nonlinear energy sinks," Nonlinear Dynamics, vol. 79, no. 1, pp. 527-538, 2015.

[26] J. Mayet and H. Ulbrich, "First-order optimal linear and nonlinear detuning of centrifugal pendulum vibration absorbers," Journal of Sound and Vibration, vol. 335, pp. 34-54, 2015.

[27] A. G. Haddow and S. W. Shaw, "Centrifugal pendulum vibration absorbers: an experimental and theoretical investigation," Nonlinear Dynamics, vol. 34, no. 3, pp. 293-307, 2003.

[28] J. S. Issa and S. W. Shaw, "Synchronous and non-synchronous responses of systems with multiple identical nonlinear vibration absorbers," Journal of Sound and Vibration, vol. 348, pp. 105-125, 2015.

[29] K. Nishimura, T. Ikeda, and Y. Harata, "Localization phenomena in torsional rotating shaft systems with multiple centrifugal pendulum vibration absorbers," Nonlinear Dynamics, vol. 83, no. 3, pp. 1705-1726, 2016.

[30] B. J. Vidmar, B. F. Feeny, S. W. Shaw, A. G. Haddow, B. K. Geist, and N. J. Verhanovitz, "The effects of Coulomb friction on the performance of centrifugal pendulum vibration absorbers," Nonlinear Dynamics, vol. 69, no. 1-2, pp. 589-600, 2012.

[31] C. Shi and R. G. Parker, "Modal properties and stability of centrifugal pendulum vibration absorber systems with equally spaced, identical absorbers," Journal of Sound and Vibration, vol. 331, no. 21, pp. 4807-4824, 2012.

[32] C. Shi and R. G. Parker, "Modal structure of centrifugal pendulum vibration absorber systems with multiple cyclically symmetric groups of absorbers," Journal of Sound and Vibration, vol. 332, no. 18, pp. 4339-4353, 2013.

[33] C. Shi, S. W. Shaw, and R. G. Parker, "Vibration reduction in a tilting rotor using centrifugal pendulum vibration absorbers," Journal of Sound and Vibration, vol. 385, pp. 55-68, 2016.

[34] A. Muszynska, Rotordynamics, CRC Press, Taylor \& Francis Group, Boca Raton, FL, USA, 2005.

[35] W. Kim, J. Y. Lee, and J. Chung, "Dynamic analysis for a planetary gear with time-varying pressure angles and contact ratios," Journal of Sound and Vibration, vol. 331, no. 4, pp. 883-901, 2012.

[36] H. Ma, H. Li, H. Niu, R. Song, and B. Wen, "Numerical and experimental analysis of the first-and second-mode instability in a rotor-bearing system," Archive of Applied Mechanics, vol. 84, no. 4, pp. 519-541, 2014. 


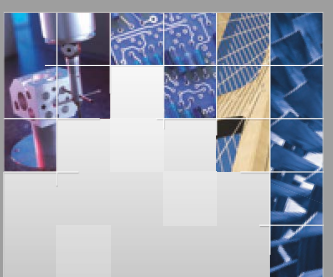

\section{Enfincering}
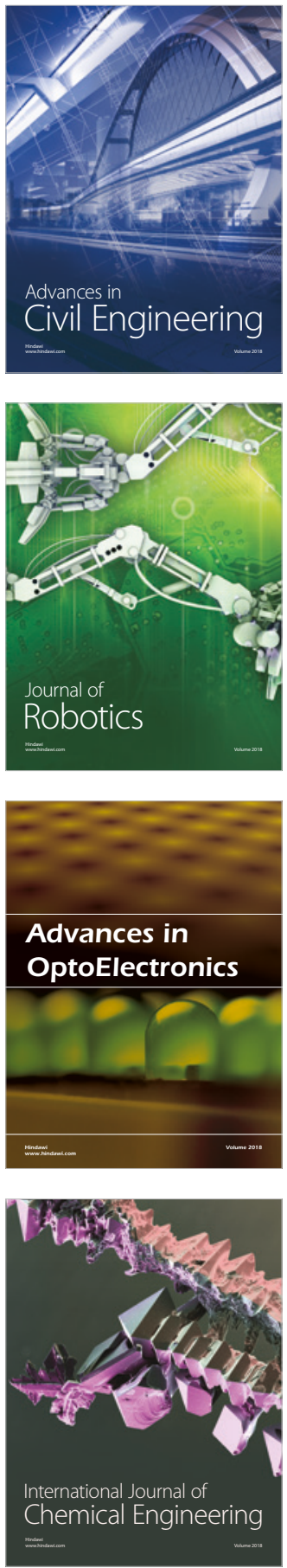

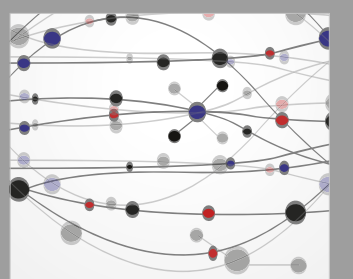

\section{Rotating \\ Machinery}

The Scientific World Journal

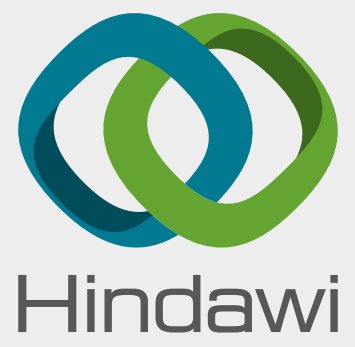

Submit your manuscripts at

www.hindawi.com
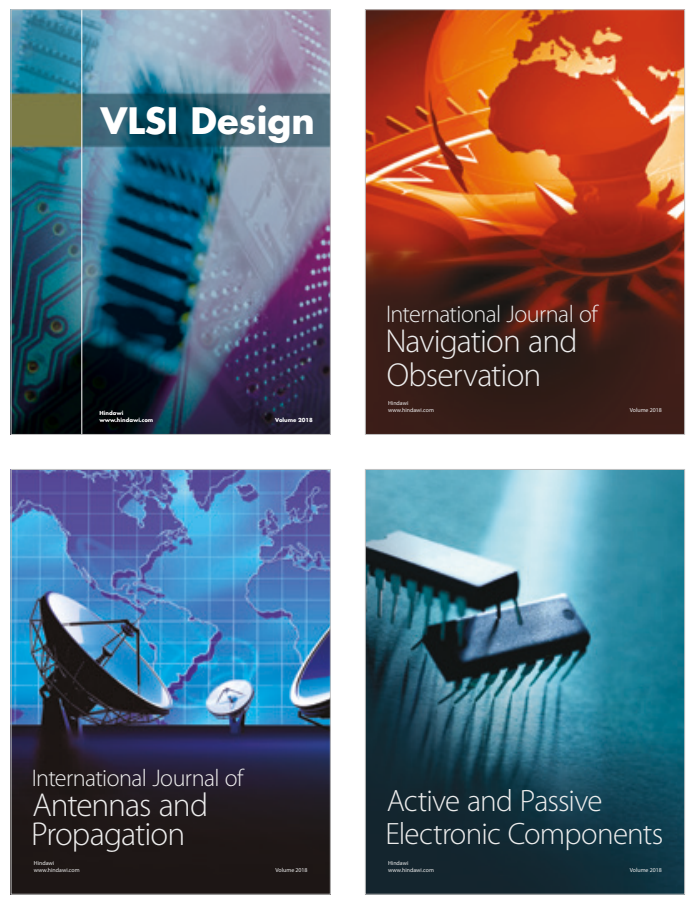
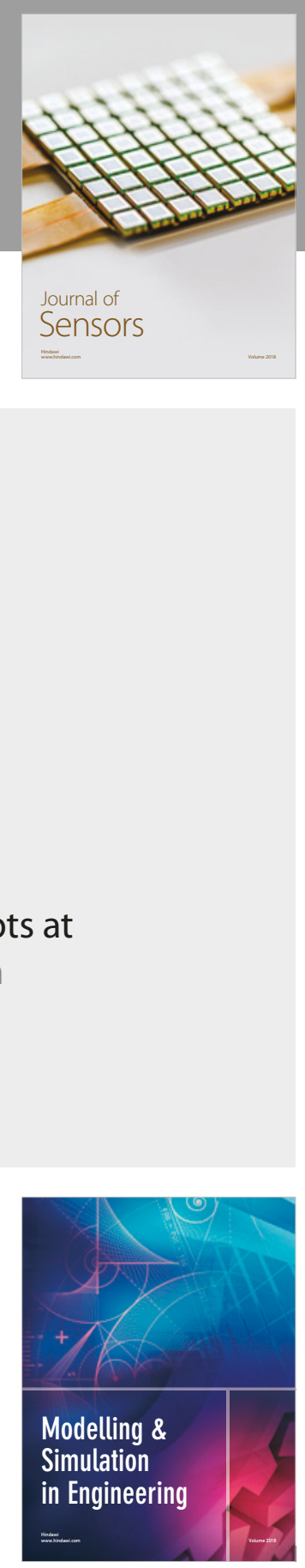

\section{Advances \\ Multimedia}
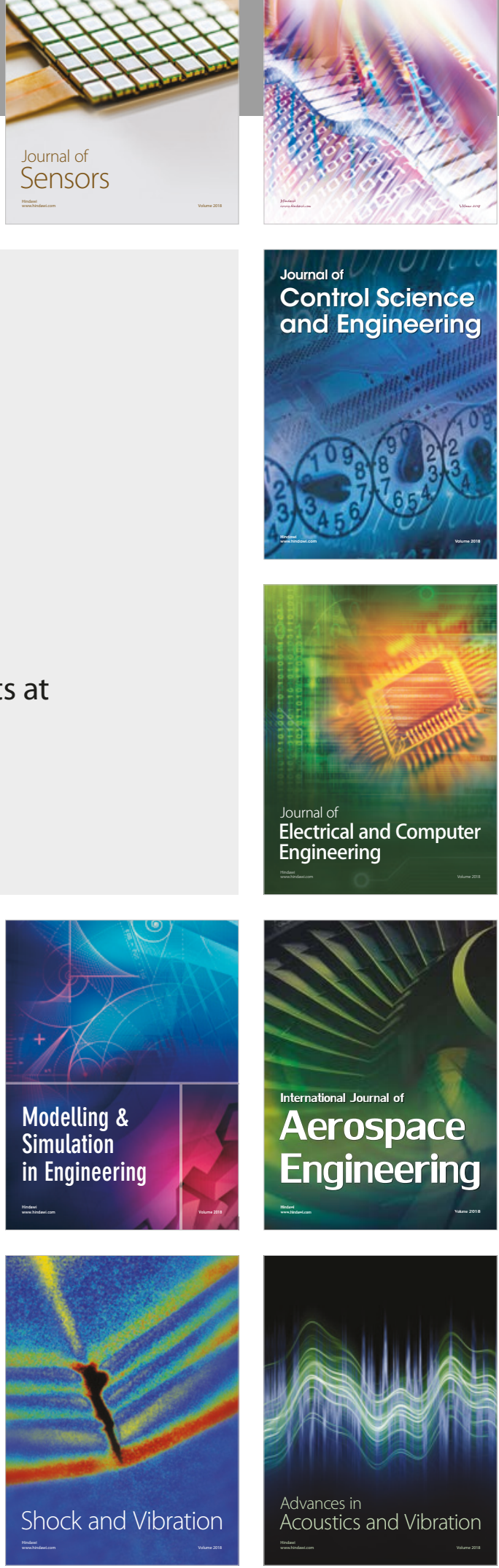